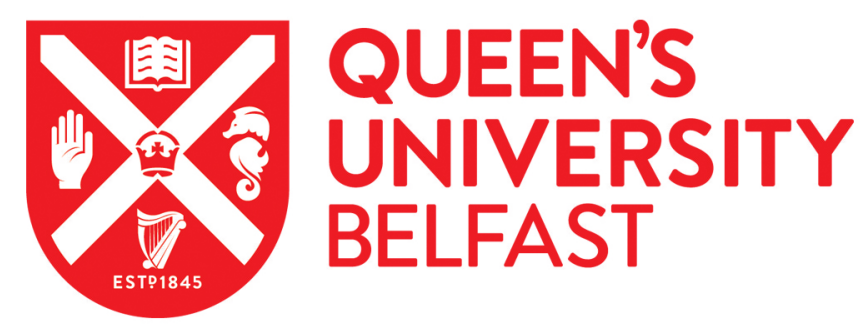

\title{
Joint Optimisation of Real-time Deployment and Resource Allocation for UAV-Aided Disaster Emergency Communications
}

Do-Duy, T., Nguyen, L. D., Duong, T. Q., Khosravirad, S., \& Claussen, H. (2021). Joint Optimisation of Real-time Deployment and Resource Allocation for UAV-Aided Disaster Emergency Communications. IEEE Journal on Selected Areas in Communications. https://doi.org/10.1109/JSAC.2021.3088662

Published in:

IEEE Journal on Selected Areas in Communications

Document Version:

Peer reviewed version

Queen's University Belfast - Research Portal:

Link to publication record in Queen's University Belfast Research Portal

Publisher rights

Copyright 2021, IEEE.

This work is made available online in accordance with the publisher's policies. Please refer to any applicable terms of use of the publisher.

\section{General rights}

Copyright for the publications made accessible via the Queen's University Belfast Research Portal is retained by the author(s) and / or other copyright owners and it is a condition of accessing these publications that users recognise and abide by the legal requirements associated with these rights.

Take down policy

The Research Portal is Queen's institutional repository that provides access to Queen's research output. Every effort has been made to ensure that content in the Research Portal does not infringe any person's rights, or applicable UK laws. If you discover content in the Research Portal that you believe breaches copyright or violates any law, please contact openaccess@qub.ac.uk. 


\title{
Joint Optimisation of Real-time Deployment and Resource Allocation for UAV-Aided Disaster Emergency Communications
}

\author{
Tan Do-Duy, Long D. Nguyen, Trung Q. Duong^, Saeed Khosravirad, and Holger Claussen
}

\begin{abstract}
In this work, we consider a joint optimisation of real-time deployment and resource allocation scheme for UAVaided relay systems in emergency scenarios such as disaster relief and public safety missions. In particular, to recover the network within a disaster area, we propose a fast K-meansbased user clustering model and jointly optimal power and time transferring allocation which can be applied in the real system by using UAVs as flying base stations for real-time recovering and maintaining network connectivity during and after disasters. Under the stringent QoS constraints, we then provide centralised and distributed models to maximise the energy efficiency of the considered network. Numerical results are provided to illustrate the effectiveness of the proposed computational approaches in terms of network energy efficiency and execution time for solving the resource allocation problem in real-time scenarios. We demonstrate that our proposed algorithm outperforms other benchmark schemes.
\end{abstract}

Index Terms-UAV cellular communication, K-means clustering, convex optimisation, embedded programming, disaster communication.

\section{INTRODUCTION}

In a disaster recovery and relief scenario, such as aftermath of flooding, hurricanes and earthquakes, traditional terrestrial cellular networks are likely to be disrupted due to failure of part of the base station (BS) infrastructure [2]-[4]. Therefore, there is a demand for the persistent and reliable communication networks that supports data transmission in disaster regions. Several technical solutions have been proposed as

The work of Tan Do-Duy belongs to the project in 2021 funded by Ho Chi Minh City University of Technology and Education, Vietnam. The work of L. D. Nguyen and T. Q. Duong was supported in part by the Global Challenges Research Fund under the DfE-GCRF 2020-2021 scheme between Queen's University Belfast, UK and Dong Nai University, Vietnam. The work of T. Q. Duong was supported in part by the U.K. Royal Academy of Engineering (RAEng) under the RAEng Research Chair and Senior Research Fellowship scheme Grant RCSRF2021 \11 \41.

Tan Do-Duy is with the Department of Computer and Communications Engineering, HCMC University of Technology and Education, 700000 Vietnam (email: tandd@hcmute.edu.vn).

Long D. Nguyen is with the Department of Engineering, Dong Nai University, Bien Hoa, Dong Nai 810000, Vietnam (email:dinhlonghcmut@gmail.com).

Trung Q. Duong is with the School of Electronics, Electrical Engineering and Computer Science, Queen's University Belfast, Belfast, BT7 1NN, UK (email:trung.q.duong@qub.ac.uk).

Saeed R. Khosravirad is with Nokia Bell Labs, Murray Hill, NJ 07964 USA (e-mail: saeed.khosravirad@nokia-bell-labs.com).

Holger Claussen is with Tyndall National Institute, Cork, T12 R5CP, Ireland, (e-mail: holger.claussen@tyndall.ie).

* Corresponding author is Trung Q. Duong. This paper has been presented in part at IEEE International Workshop on Signal Processing Advances in Wireless Communications (SPAWC), Cannes, France, July 2019 [1]. potential candidates for solving the problem. For example, device-to-device (D2D) networks can be used to transmit packets between nodes in proximity without traversing the BSs or core network [5]. However, it is very challenging to optimize the routes between distributed nodes in disaster areas due to adverse wireless channels. In another attempt, satellite communications might be a potential technology in emergency communications as it can be used in disaster areas where the terrestrial networks are not available [6], yet limited and costly resources (low data rates and high end-to-end latency) and limited equipment available to most public users are main obstacles to the wide deployment of satellites. By providing deployment flexibility and mobility with adaptive altitude, unmanned aerial vehicle (UAV) based cellular network has been recently considered as a key solution to support reliable communication services to ground users (UEs) at a particular location in the disaster area [7]-[9]. As compared to the conventional technologies, the main advantage of using UAVs as flying and altitude-adjustable relay nodes is to exploit the line-of-sight (LoS) communication links to multiple UEs [10], [11].

The efficient UAV deployment problem in wireless networks has been extensively studied to improve network performance. For instance, in [12], the authors investigated the efficient solutions for synchronization and data management in a UAVassisted emergency Wi-Fi network over the disaster area. The work in [13] focused on the optimal altitude and location of multiple UAVs working as flying BSs to maximize the coverage. Most recently, considering UAV-aided communications, the work [14] investigated a joint UAV-user association and UAV deployment location optimization problem, yet the main objective in [14] is to maximize users' total achievable rates. In other works [15], [16], an end-to-end throughput maximization problem was proposed to optimize UAV-based relay trajectory and power allocation. In addition, the authors in [17] studied energy-efficient UAV trajectory path planning for a UAV-based relay network to serve ground nodes. However, the paper [14] [17] only considered a simple network including a source node and a single UAV-assisted relay node. Furthermore, in [18], the trajectory design problem was investigated for a UAV-enabled multicasting system considering UAV mission completion time to ensure some required successful packet recovery probability at the ground nodes. Nevertheless, in general, there is a lack of practical solutions for joint optimal UAV deployment and resource allocation considering the real-time emergency scenarios in UAV-enabled cellular networks. Very recently, the 
joint optimisation for UAV's clustering selection and resource allocation has been considered in [1]. In particular, the target of the work in [1] is to maximise the network throughput via UAVs clustering selection and power allocation, i.e., optimal max-min end-to-end (E2E) rate of each user. However, such joint optimisation in maximising the throughput exploits the full power allocation, which neglects the energy efficiency (EE) of the whole networks and therefore, is inapplicable to disaster emergency communications.

Moreover, the conventional K-means algorithm proposed in [19], a method for clustering approach, may not be suitable for UAVs with the stringent QoS requirements. Due to the dynamic nature of UAV communications with complex procedures, the current version of K-means method may not be suitable for UAV with many constraints, which cannot be directly applied to our work. Therefore, in this paper, we propose a new K-means clustering model with sophisticated QoS constraints for solving the UAV deployment. Thus, different from the aforementioned works, in this paper, we propose a real-time optimisation framework for the practical and simultaneous deployment of multiple UAVs in disaster relief networks under stringent quality-of-services (QoS) requirement and energy constraints. In particular, the main contributions of this paper can be summarized as follows:

- We propose a fast and efficient user clustering model with QoS constraints based on a popular user clustering method, called K-means clustering [19], to form multiple clusters of distributed UEs within the disaster area who cannot be directly served by the still operating cellular BSs due to poor wireless channel conditions. Then, a central station deploys a number of UAVs as flying relay nodes at the centroid of clusters to support communication services to the set of UEs in each cluster.

- Under the stringent QoS requirements of disaster emergency communications and energy-efficiency constraints of UAV-aided relay systems, we develop and solve a distributed real-time resource allocation problem for maximising $\mathrm{EE}$ in terms of the ratio between overall transmitted data to all users in the disaster area and total energy consumption via joint UAVs trajectory planning, power and time allocation.

- By means of numerical results and implementation in embedded systems using Python programming, we demonstrate the effectiveness of the proposed approach for solving the practical resource allocation of the UAV-aided communication systems.

The paper is organized as follows. After the Introduction, the system model is presented in Section II which is used throughout this paper and the main optimization problems to be solved in the remaining sections. The UAV deployment by optimal UAV-UE clustering model corresponding to the QoS constraints is investigated in Section III. The UAV-enabled disaster emergency communications via central resource allocation including maximising network EE performance with EE objective and enhancing network EE performance with fairness services is presented in Section IV. On the other hand, the distributed resource allocation model for UAV- enabled disaster emergency communications is discussed in Section V. Extensive numerical results and implementation in embedded systems using Python programming to indicate the performance of the proposed approaches are conducted in Section VI. Finally, the main contributions and conclusions of the paper are provided in Section VII.

\section{SYSTEM MODEL AND PROBLEM FORMULATION}

\section{A. System model}

We consider the downlink transmission where a massive MIMO BS is equipped with a large $L$-antenna array to serve some low-mobility UEs in a safety area. Meanwhile, $K$ UEs, whose locations are randomly distributed in the disaster area, are grouped into $M$ clusters. To support $M$ clusters of UEs which are isolated in the disaster region, $M$ UAVs, $\mathcal{M}=\{1, \ldots, M\}$, are deployed to connect the UEs with the BS. The UAVs and UEs are equipped with a single antenna. The cluster $m$ can serve a finite number of UEs, $\mathcal{K}_{m}=\left\{1, \ldots, K_{m}\right\}$ for $m \in \mathcal{M}$. The $(m, k)$ th UE denotes the $k$ th user in the $m$ th cluster. A typical model of cellularconnected UAV communication in disaster relief is illustrated in Fig. 1. Here, UAVs play the vital role of flying relay stations (RSs) to provide reliable communication between a BS and a large number of distributed users in a disaster region. In such a scenario, the UAV deployment is a key design consideration due to its stringent energy and placement constraints. In addition, the flying UAV-based RSs need to reorganize and selfdeploy to cope with the dynamically changing environment in disaster areas. Hence, decision-making for self-organization and resource allocation should be realized in real time [4], [8], [9] i.e., in the time scales of milliseconds [20].

\section{B. Channel model}

We consider that all the UAVs operate over a time duration $T>0$ (in seconds). The time-varying 3D locations of the BS, the $m$ th UAV and the $k$ th user $(m=1, . ., M$ and $k=1, \ldots, K)$ are denoted by $\left(x_{0}, y_{0}, H_{0}\right)$ and $\left(x_{U, m}(t), y_{U, m}(t), H_{U, m}(t)\right)$, and $\left(x_{k}, y_{k}, 0\right)$, respectively. Here, $H_{0}$ and $H_{U, m}(t)$ denote the antenna heights of the BS and the UAV, respectively. We assume that the antenna altitude of the UAV is also the altitude of the UAV.

Without loss of generality, the time duration $T$ is divided into $N$ equal time-slots. Hence, the length of each time-slot is given as $\gamma=\frac{T}{N}$, which is assumed to be sufficiently small such that the location of the UAV is stable within a time-slot [18]. Therefore, the trajectory of the $m$ th UAV with respect to the $n$th time-slot $(n=1, \ldots, N)$ can be approximated as $q_{m}[n]=\left(x_{U, m}[n], y_{U, m}[n], H_{U, m}[n]\right)$.

The trajectory of the UAV is assumed to be contained within the considered area. To start the mission, the UAVs fly from their parking dock at the location $\boldsymbol{q}_{G, m}=\left[x_{G, m}, y_{G, m}, h_{G, m}\right]$, i.e., $\boldsymbol{q}_{m}[1]=\boldsymbol{q}_{G, m}$, to the disaster area and relay the information data from the BS to the allocated UEs, and finish their trajectories at their parking dock, i.e., $\boldsymbol{q}_{m}[N]=\boldsymbol{q}_{G, m}$.

At the $n$th time-slot, the distance between the BS and the $m$ th UAV is written as

$$
R_{0, m}[n]=\sqrt{d_{0, m}^{2}[n]+\left(H_{U, m}[n]-H_{0}\right)^{2}},
$$




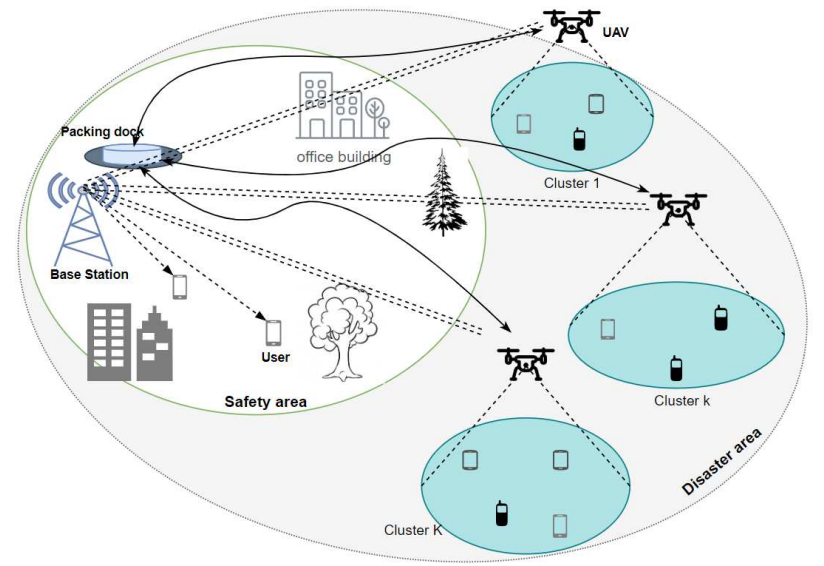

Fig. 1: A typical model of cellular-connected UAV communication in disaster relief [1].

where $d_{0, m}[n]=\sqrt{\left(x_{U, m}[n]-x_{0}\right)^{2}+\left(y_{U, m}[n]-y_{0}\right)^{2}}$.

Similarly, the distance between the $m$ th UAV and the $k$ th user in the $m$ th cluster is given by

$$
R_{m, k}[n]=\sqrt{d_{m, k}^{2}[n]+H_{U, m}^{2}[n]}, k \in \mathcal{K}_{m},
$$

where $d_{m, k}[n]=\sqrt{\left(x_{U, m}[n]-x_{k}\right)^{2}+\left(y_{U, m}[n]-y_{k}\right)^{2}}$ is the Euclidean distance between the $m$ th UAV and the $k$ th user.

Due to the high altitude of the UAV, we assume that the airto-air (ATA) channel between the BS and the UAVs is LoS. Hence, the path loss (in $\mathrm{dB}$ ) between the BS and the $m$ th UAV is only dependent on free-space path loss and is modeled as

$$
\begin{aligned}
\beta_{0, m}[n] & =10 \log \left(\beta_{0} R_{0, m}^{-2}[n]\right) \\
& =10 \log \left(\frac{\beta_{0}}{d_{0, m}^{2}[n]+\left(H_{U, m}[n]-H_{0}\right)^{2}}\right),
\end{aligned}
$$

where $\beta_{0}$ is the channel power gain at reference distance $d_{0}$.

In contrast, the air-to-ground (ATG) channel between the UAVs and the users has a more complex form, due to the impacts of the environment, including shadowing from the propagation blockages, which can result in a NLoS channel. The path loss (in $\mathrm{dB}$ ) between the $m$ th UAV and the $k$ th user is calculated as [13]

$$
\begin{aligned}
\beta_{m, k}[n] & =P L_{m, k}[n]+\eta^{\operatorname{LoS}} P_{m, k}^{\operatorname{LoS}}[n]+\eta^{N o S} P_{m, k}^{N \operatorname{LoS}}[n] \\
& =10 \alpha \log \left(\sqrt{d_{m, k}^{2}[n]+H_{U, m}^{2}[n]}\right)+A P_{m, k}^{\operatorname{LoS}}[n]+B,
\end{aligned}
$$

where $\eta^{L o S}$ and $\eta^{N L o S}$ are the average additional losses for LoS and NLoS, respectively. In addition, we have

$A=\eta^{L o S}-\eta^{N L o S}, B=10 \alpha \log \left(\frac{4 \pi f_{c} R_{m, k}[n]}{c}\right)+\eta^{N L o S}$.

Therein, the distance path loss is given by

$$
P L_{m, k}[n]=10 \log \left(\frac{4 \pi f_{c} R_{m, k}[n]}{c}\right)^{\alpha},
$$

where $f_{c}$ is carrier frequency $(\mathrm{Hz}), c$ is the speed of light $(\mathrm{m} / \mathrm{s})$, $\alpha \geq 2$ is the path loss exponent. The probability of $\operatorname{LoS}$ and NLoS is given by [21]

$$
\begin{aligned}
P_{m, k}^{\operatorname{LoS}}[n] & =\frac{1}{1+a \exp \left[-b\left(\arctan \left(\frac{H_{U, m}[n]}{d_{m, k}[n]}\right)-a\right)\right]}, \\
P_{m, k}^{N \operatorname{LoS}}[n] & =1-P_{m, k}^{\operatorname{LoS}[n],}
\end{aligned}
$$

where the constants $a$ and $b$ depend on environment. On the other hand, the small-scale fading of all channels $\left(\boldsymbol{h}_{0, m}[n] \in\right.$ $\left.\mathbb{C}, h_{m, k}[n] \in \mathbb{C}\right)$ is assumed as an independent and identically distributed (i.i.d.) random variable with zero mean and unit variance. For ease of readability, in the next sections, we will omit the discrete time-domain $([n])$ from mathematical notations and expressions.

\section{Transmission model}

We consider the two-phase communication of downlink transmission. In the first phase, the BS transmits the signal to the UAVs. The signal received at the $m$ th UAV is given by

$$
\begin{aligned}
y_{0, m}= & \underbrace{\boldsymbol{g}_{0, m}^{H} \sqrt{P_{0}} \boldsymbol{f}_{0, m} x_{0, m}}_{\text {desired signal }} \\
& +\underbrace{\sum_{m^{\prime} \in \mathcal{M} \backslash\{m\}} \boldsymbol{g}_{0, m^{\prime}}^{H} \sqrt{P_{0}} \boldsymbol{f}_{0, m^{\prime}} x_{0, m^{\prime}}}_{\text {co-tier interference }}+n_{m},
\end{aligned}
$$

where $\boldsymbol{g}_{0, m} \in \mathbb{C}^{L}$ is the channel coefficient between the BS and $m$ th UAV. Here, we utilize the structure of the ATA by including both large-scale and small-scale fading effects as $\boldsymbol{g}_{0, m}=\sqrt{\beta_{0, m}} \boldsymbol{h}_{0, m}$, where $\boldsymbol{h}_{0, m}$ is the small-scale fading coefficient for channel from BS to $m$ th UAV. In addition, $\boldsymbol{f}_{0, m}$ is the vector beamforming and $x_{0, m} \in \mathbb{C}$ is the information received at the $m$ th UAV with $\left\|x_{0, m}\right\|^{2} \leq 1 ; P_{0}$ is the transmit power at the $\mathrm{BS} ; n_{m} \sim \mathcal{C N}\left(0, \sigma_{m}^{2}\right)$ is the additive white Gaussian noise (AWGN) at the $m$ th UAV.

We assume that the BS uses a channel estimation process to estimate the effective channels to design the precoding matrix. In this paper, we employ the maximal ratio transmission (MRT), which is a simple and nearly optimal precoding design in massive MIMO networks [22]. The MRT downlink precoders at the BS and the UAV are given by

$$
\boldsymbol{f}_{0, m}=\sqrt{p_{0, m}} \frac{\boldsymbol{g}_{0, m}^{*}}{\left\|\boldsymbol{g}_{0, m}\right\|},
$$

where $p_{0, m}$ is power control coefficient. In the second phase, the UAVs forward signals to the users in their cluster. The signal received by the UE $k$ in cluster $m$ is written as

$$
\begin{aligned}
y_{m, k}= & \underbrace{g_{m, k} \sqrt{P_{m}} \sqrt{p_{m, k}} x_{m, k}}_{\text {desired signal }} \\
& +\underbrace{\sum_{l \in \mathcal{M} \backslash\{m\}} \sum_{j \in \mathcal{K}_{l}} g_{l, k_{m}} \sqrt{P_{l}} \sqrt{p_{l, j}} x_{l, j}}_{\text {inter-cluster interference }}+n_{m, k},
\end{aligned}
$$

where $g_{m, k} \in \mathbb{C}$ is the channel between the $m$ th UAV and the $k$ th UE. Similarly as in ATA interface, for ATG channel, 
we also have $g_{m, k}=\sqrt{\beta_{m, k}} h_{m, k}$, where $h_{m, k}$ is the smallscale fading coefficient for the channel from $m$ th UAV to $k$ th user. Moreover, $P_{m}$ is the transmit power at the $m$ th UAV ${ }^{1}$, $p_{m, k}$ and $x_{m, k}$ are the power control coefficient and received information signal at the $k$ th $\mathrm{UE}$ in the $m$ th cluster with $\left\|x_{m, k}\right\|^{2} \leq 1$, and $n_{m, k} \sim \mathcal{C N}\left(0, \sigma_{m, k}^{2}\right)$ is AWGN at the $k$ th UE.

Given the power control coefficients $\boldsymbol{p}_{0}=\left[p_{0, m}\right]_{m=1}^{M}, \boldsymbol{p}_{m}=$ $\left[p_{m, k}\right]_{k=1}^{K_{m}}$, the network interference is characterized by the cotier interference power in the first phase as

$$
\mathcal{I}_{m}^{\text {cotier }}\left(\boldsymbol{p}_{0}\right)=\sum_{m^{\prime} \in \mathcal{M} \backslash\{m\}} P_{0} p_{0, m^{\prime}} \gamma_{0, m, m^{\prime}}, m \in \mathcal{M},
$$

where $\gamma_{0, m, m^{\prime}}=\left|\left\langle\boldsymbol{g}_{0, m^{\prime}}, \frac{\boldsymbol{g}_{0, m^{\prime}}^{*}}{\left\|\boldsymbol{g}_{0, m^{\prime}}\right\|}\right\rangle\right|^{2},\langle$.$\rangle is the Hermitian$ inner product, and the inter-cluster interference functions ${ }^{2}$ in the second phase, written as

$$
\mathcal{I}_{m, k}^{\text {inter }}\left(\boldsymbol{p}_{M}\right)=\sum_{l \in \mathcal{N}_{m}} \sum_{j \in \mathcal{K}_{l}} \beta_{l, k} P_{l} p_{l, j}, k \in \mathcal{K}_{m} ; m \in \mathcal{M},
$$

where $\boldsymbol{p}_{M}=\left[\boldsymbol{p}_{m}\right]_{m=1}^{M}$ and $\mathcal{N}_{m} \subset \mathcal{M} \backslash\{m\}$.

The information throughput in the first phase at the $m$ th $\mathrm{UAV}$ (in nats) is shown as

$$
R_{0, m}\left(\boldsymbol{p}_{0}\right)=\frac{1}{2} B \ln \left(1+\frac{P_{0} p_{0, m} \gamma_{0, m, m}}{\mathcal{I}_{m}^{\operatorname{cotier}}\left(\boldsymbol{p}_{0}\right)+\sigma_{m}^{2}}\right),
$$

where $B$ is the bandwidth of the system, $\gamma_{0, m, m}=$ $\left|\left\langle\boldsymbol{g}_{0, m}, \frac{\boldsymbol{g}_{0, m}^{*}}{\left\|\boldsymbol{g}_{0, m}\right\|}\right\rangle\right|^{2}$.

The information throughput in the second phase at the $(m, k)$ th UE (in nats) is written as

$$
R_{m, k}\left(\boldsymbol{p}_{M}\right)=\frac{1}{2} B \ln \left(1+\frac{P_{m} p_{m, k}\left|g_{m, k}\right|^{2}}{\mathcal{I}_{m, k}^{\text {inter }}\left(\boldsymbol{p}_{M}\right)+\sigma_{m, k}^{2}}\right) .
$$

Then, E2E information throughput at the $(m, k)$ th $\mathrm{UE}$ is given by

$$
R_{m, k}^{E 2 E}\left(\boldsymbol{p}_{0}, \boldsymbol{p}_{M}\right)=\min \left\{R_{0, m}\left(\boldsymbol{p}_{0}\right), R_{m, k}\left(\boldsymbol{p}_{M}\right)\right\} .
$$

Hence, the transmission data (in bits) received by the UE $(m, k)$ is given by

$$
\begin{aligned}
D_{m, k}^{E 2 E}\left(\boldsymbol{p}_{0}, \boldsymbol{p}_{M}, t_{m, k}^{d a t}\right) & =R_{m, k}^{E 2 E}\left(\boldsymbol{p}_{0}, \boldsymbol{p}_{M}\right) t_{m, k}^{d a t} \\
& =\min \left\{R_{0, m}\left(\boldsymbol{p}_{0}\right) t_{m, k}^{d a t}, R_{m, k}\left(\boldsymbol{p}_{M}\right) t_{m, k}^{d a t}\right\},
\end{aligned}
$$

where $t_{m, k}^{d a t}$ is the data transferring time for the $m$ th UAV and $(m, k)$ th UE exchange duration. In this work, we use the time division access scheme, so the intra-cluster interference can be fully suppressed.

\footnotetext{
${ }^{1}$ We assume that the transmit power $P_{m}$ is from an energy source that is separate from the UAV's internal battery and is used to power the UAV operation. We further assume that this energy source is much larger than the UAV's internal battery.

${ }^{2}$ The inter-cell channel $h_{l, k}$ is difficult to estimate, and thus, it must be defined as in (12).
}

Then, the total transmission data received by all the UEs in the deployment area can be expressed as

$$
D_{t o t}^{E 2 E}\left(\boldsymbol{p}_{0}, \boldsymbol{p}_{M}, \tau_{M}^{d a t}\right)=\sum_{m \in \mathcal{M}} \sum_{k \in \mathcal{K}_{m}} D_{m, k}^{E 2 E}\left(\boldsymbol{p}_{0}, \boldsymbol{p}_{M}, t_{m, k}^{d a t}\right),
$$

where $\mathbf{t}_{m}^{d a t}=\left[t_{m, k}^{d a t}\right]_{k=1}^{K_{m}}$ and $\tau_{M}^{d a t}=\left[\mathbf{t}_{m}^{d a t}\right]_{m=1}^{M}$.

Unlike [1] where the main goal is to maximise the minimum throughput of all clusters, i.e., maxmin E2E rate problem, in this paper, we take a step further to tackle the network energy efficiency (EE) which is more relevant to the perspective of UAV communications. As such, we will detail the time and energy models with respect to the context of UAV communications in the next sub-sections.

\section{Energy modeling with time framework}

The total operating time of the $m$ th UAV is estimated by

$$
t_{m}^{o p e}\left(\boldsymbol{q}_{m}, \mathbf{t}_{m}^{\text {dat }}\right)=\sum_{k \in \mathcal{K}_{m}} t_{m, k}^{c o n}+\sum_{k \in \mathcal{K}_{m}} t_{m, k}^{d a t}+t_{G, m}^{f l y}+t_{m, G}^{f l y},
$$

where $\sum_{k \in \mathcal{K}_{m}} t_{m, k}^{c o n}$ and $\sum_{k \in \mathcal{K}_{m}} t_{m, k}^{d a t}$ are the connecting time and the data transferring time for each exchange duration of the $m$ th cluster, respectively. Here, $t_{G, m}^{f l y}$ and $t_{m, G}^{f l y}$ denote the flying duration of the $m$ UAV from the parking dock to the centroid of the $m$ th cluster and vice versa.

We introduce operating time limitation of the UAVs

$$
t_{m}^{\text {ope }} \leq T_{m}^{\max }, m \in \mathcal{M}
$$

where $T_{m}^{\max }$ is the maximum operating time of the $m$ th UAV.

The total energy (in Joules) consumed by the BS and all UAVs is defined by

$$
\begin{aligned}
& \vartheta_{t o t}\left(\boldsymbol{p}_{0}, \boldsymbol{p}_{M}, M, \boldsymbol{q}_{M}, \tau_{M}^{d a t}\right)=\eta_{0} P_{0} \sum_{m \in \mathcal{M}} p_{0, m} \sum_{k \in \mathcal{K}_{m}} t_{m, k}^{d a t} \\
& +\sum_{m \in \mathcal{M}}\left(t_{m}^{\text {ope }}\left(\boldsymbol{q}_{m}, \mathbf{t}_{m}^{\text {dat }}\right) P_{m}^{\text {fly }}+\eta_{m} P_{m} \sum_{k \in \mathcal{K}_{m}} p_{m, k} t_{m, k}^{d a t}\right)
\end{aligned}
$$

where $\eta_{0}, \eta_{m}>0$ are the reciprocal drain efficiency of the power amplifier at the BS and UAVs; $P_{m}^{f l y}$ is the power consumption of the $m$ th UAV for flying duration.

\section{E. Problem formulation}

In this paper, we aim at maximising the network EE, which is described as the ratio between overall transmitted data to all UEs in the disaster area and total energy consumption via UAV's trajectory planning and clustering selection model 
and power allocation. To this end, the explicit EE problem is formulated as

$$
\begin{aligned}
\max _{\boldsymbol{p}_{0}, \boldsymbol{p}_{M}, M, \boldsymbol{q}_{M}, \tau_{M}^{\text {dat }}} & \frac{D_{\text {tot }}^{E 2 E}\left(\boldsymbol{p}_{0}, \boldsymbol{p}_{M}, \tau_{M}^{\text {dat }}\right)}{\vartheta_{\text {tot }}\left(\boldsymbol{p}_{0}, \boldsymbol{p}_{M}, M, \boldsymbol{q}_{M}, \tau_{M}^{\text {dat }}\right)} \\
\text { subject to } & (19), \\
& \sum_{m \in \mathcal{M}} p_{0, m} \leq 1, \sum_{k \in \mathcal{K}_{m}} p_{m, k} \leq 1, m \in \mathcal{M}, \\
& R_{0, m}\left(\boldsymbol{p}_{0}\right) \geq \bar{r}_{0, m}, m \in \mathcal{M}, \\
& \boldsymbol{q}_{m i n} \leq \boldsymbol{q}_{m} \leq \boldsymbol{q}_{\text {max }}, \\
& \boldsymbol{q}_{M}=\left[\boldsymbol{q}_{m}\right]_{m=1}^{m=M}, m \in \mathcal{M}, \\
& (m, k) \in \mathcal{K}_{m}, m \in \mathcal{M},
\end{aligned}
$$

where $(21 \mathrm{~b})$ represents the operating time constraint of the UAVs, (21c) represents the power constraints at the BS and the UAVs, (21d) sets the QoS requirement in BS-UAV links at the first phase, (21e) stands for the trajectory constraints of the UAVs, and (21f) is the cluster selection model. As can be seen from (21), the optimisation problem is a non-convex problem with the non-convex functions of (21a), (21d) and (21f). Moreover, for large-scale scenarios, the problem (21) becomes very complex due to the large number of timeslots $(N)$, and large number of UAVs $(M)$ and UEs $(K)$ deployed in the area.

To efficiently solve the above non-convex problem in realtime scenarios, we separate problem (21) into two subproblems. Firstly, the UAV-UE clustering method with QoS constraints and UAVs trajectory planning will be proposed for the constraints (21e)-(21f) by the constrained K-means clustering procedure to minimize the number of UAVs. This will be discussed in detail in Section III. After the completion of the UAVs' deployment, a resource allocation algorithm is implemented to maximise the EE with the operating time constraint (21b) and the constrained power budget (21c). In this paper, we propose two distinct optimal resource allocation algorithms in a centralised and distributed fashion in Section IV and Section V, respectively. For each centralised and distributed resource allocation algorithm, we propose two cases: 1) maximising the total network EE in Subsections IV-A, V-A and 2) maximising the network $\mathrm{EE}$ with fairness QoS in Subsections IV-B, V-B.

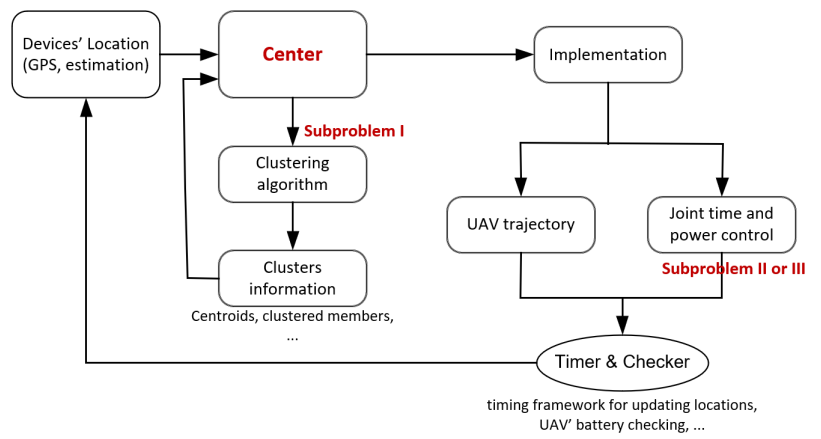

Fig. 2: Block processing operations for the proposed system model.

\section{UAV DEPLOYMENT BY OPTIMAL UAV-UE CLUSTERING MODEL}

In this section, the clustering algorithm for UAVs deployment is first implemented for supporting as many users as possible by proposing an efficient constrained K-mean clustering algorithm. Then, based on the results of the clustering algorithm, the optimal power allocation approaches in centralised and distributed manners will be discussed in the next sections.

In this paper, the requirements of a $\mathrm{K}$-means clustering are based on large-scale path loss via UEs' location data from Global Positioning System (GPS) such as the input UE's location $\boldsymbol{q}_{k}=\left(x_{k}, y_{k}\right)(k=1, \ldots, K)$ and UAV's location $\boldsymbol{q}_{m}=\left(x_{U, m}, y_{U, m}, H_{U, m}\right)(m=1, \ldots, M)$. Then, based on the clustering selection, the 3D locations of the UAVs will be chosen by the centroid $\boldsymbol{\theta}_{m}=\left(x_{m}, y_{m}, z_{m}\right), m=1, \ldots, M$. However, the conventional K-means clustering approach [19] may not be suitable for UAVs with the stringent QoS requirements, e.g, constraint (21d). In this section, we propose a constrained K-means clustering model for UAV deployment corresponding to the QoS constraints. In addition, we take into account the fact that the selected number of UAVs to form the clusters should be as small as possible due to the minimal network EE requirement. This is clearly a novel point of our clustering model compared with the previous work [1]. Specifically, in [1], the number of UAVs deployed to recover network connectivity of disaster areas was fixed because we were interested in maximising the maxmin throughput optimisation problem. In contrast, since the K-means algorithm is an unsupervised clustering, finding the optimal number of clusters (UAVs) is vitally important to network EE, which is our target in this paper.

Constrained K-means clustering is a useful way to express a priori knowledge which instances should or should not be grouped together. Thus, we provide two types of pairwise constraints as

- Must-link constraints $(m, k) \in \mathcal{C}_{\text {must }}$ indicate that the $k$ th UE has to be located in the cluster $m$ with satisfied QoS constraints.

- Cannot-link constraints $\left(m, k^{\prime}\right) \in \mathcal{C}_{\text {not }}$ imply that the $k$ th UE should not be placed in the cluster $m$.

Let $\gamma_{Q o S}$ be the path loss threshold corresponding to the QoS requirement (21d) [23]. Then, a set of must-link pairs represents the satisfied QoS constraints such that the $k$ th UE is served by the $m$ th $\mathrm{UAV}$ and $\beta_{m, k} \leq \gamma_{Q o S}$, i.e.,

$$
d_{m, k}^{2}+H_{U, m}^{2} \leq 10 \frac{\gamma_{Q o S}-\left(A P_{m, k}^{L o S}+B\right)}{10 \alpha} .
$$

In contrast, a set of cannot-link pairs represents the violation of QoS constraints. Hence, optimal UAVs deployment with respect to the minimum number of UAVs and minimum operating time, can expressed through the subproblem I as follows:

$\begin{array}{cl}\text { Subproblem I: } & \min _{\boldsymbol{q}_{M}} M \\ & \text { subject to }(21 e),(21 f),(22) .\end{array}$ 
The subproblem I is then solved by Algorithm 1 where we propose a constrained K-means clustering algorithm to identify the optimal number of clusters i.e., the minimum number of UAVs $\left(M^{*}\right)$, and the corresponding cluster members.

Furthermore, we also identify the 3D location of the UAVs as they are deployed at the centroid location of each cluster. Hence, in order to minimize the operating time, each UAV should fly in a straight line from their parking dock to the centroid location of its serving cluster and vice versa.

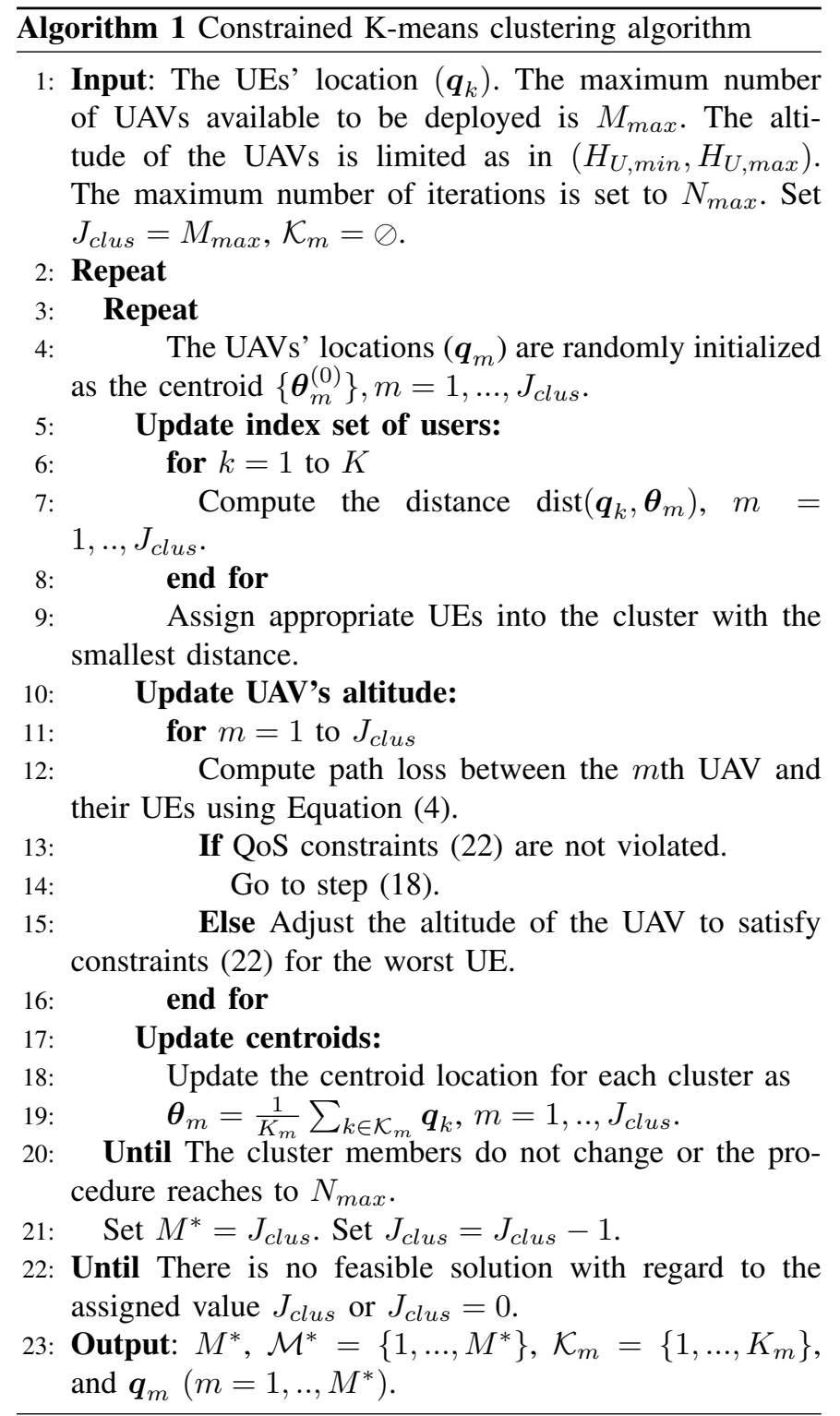

\section{COMMUNICATIONS VIA CENTRAL RESOURCE ALLOCATION}

After solving the UAV deployment problem, in this section, we present an efficient resource allocation scheme for improving network EE performance under disaster emergency communications. In this section, we consider a central computing platform for problem solving.
A. A central model for maximising network EE performance with EE objective (CEE-EE)

In this subsection, we are interested in a central model for maximising network EE performance with EE objective (CEE-EE). Here, we modify the original problem (21) by investigating EE maximization problem as

Subproblem II - A : $\max _{\boldsymbol{p}_{0}, \boldsymbol{p}_{M}, \tau_{M}^{d a t}} \frac{D_{t o t}^{E 2 E}\left(\boldsymbol{p}_{0}, \boldsymbol{p}_{M}, \tau_{M}^{d a t}\right)}{\vartheta_{t o t}\left(\boldsymbol{p}_{0}, \boldsymbol{p}_{M}, M, \boldsymbol{q}_{M}, \tau_{M}^{d a t}\right)}$

$$
\text { subject to }(19),(21 c),(21 d) \text {. }
$$

To solve the problem (24), we first change the variables as

$$
\begin{gathered}
\theta_{m, k}=\frac{1}{t_{m, k}^{\text {dat }}}, \varphi_{0, m}=\frac{1}{p_{0, m}}, \text { and } \\
\varphi_{m, k}=\frac{1}{p_{m, k}}, m \in \mathcal{M}^{*}, k \in \mathcal{K}_{m} .
\end{gathered}
$$

For $\boldsymbol{\theta}_{M}=\left\{\theta_{m, k}\right\}, \boldsymbol{\varphi}_{0}=\left\{\varphi_{0, m}\right\}, \boldsymbol{\varphi}_{M}=\left\{\varphi_{m, k}\right\}, \forall m \in$ $\mathcal{M}^{*}, k \in \mathcal{K}_{m}$, the problem (24) is equivalent to

$$
\begin{aligned}
\max _{\boldsymbol{\varphi}_{0}, \boldsymbol{\varphi}_{M}, \boldsymbol{\theta}_{M}} & \frac{D_{t o t}^{E 2 E}\left(\boldsymbol{\varphi}_{0}, \boldsymbol{\varphi}_{M}, \boldsymbol{\theta}_{M}\right)}{\vartheta_{t o t}\left(\boldsymbol{\varphi}_{0}, \boldsymbol{\varphi}_{M}, \boldsymbol{\theta}_{M}\right)} \\
\text { subject to } & \sum_{k \in \mathcal{K}_{m}} \frac{1}{\theta_{m, k}} \leq T_{m}^{\text {max }}-\left(\sum_{k \in \mathcal{K}_{m}} t_{m, k}^{c o n}+t_{G, m}^{f l y}+t_{m, G}^{f l y}\right) \\
& m \in \mathcal{M}^{*}, \\
& \sum_{m \in \mathcal{M}} \frac{1}{\varphi_{0, m}} \leq 1, \sum_{k \in \mathcal{K}_{m}} \frac{1}{\varphi_{m, k}} \leq 1, m \in \mathcal{M}^{*} \\
& R_{0, m}\left(\boldsymbol{\varphi}_{0}\right) \geq \bar{r}_{0, m}, m \in \mathcal{M}^{*},
\end{aligned}
$$

where (26b) corresponds to the operating time constraint of the UAVs after changing the variables, (26c) and (26d) represent the power constraints at the terminals, i.e., BS and the UAVs, and the constraint of QoS requirement in BS-UAV links at the first phase after the change of variables, respectively. Then, the EE objective function (26a) can be expressed as

$$
\begin{aligned}
& D_{\text {tot }}^{E 2 E}\left(\boldsymbol{\varphi}_{0}, \boldsymbol{\varphi}_{M}, \boldsymbol{\theta}_{M}\right)=\sum_{m \in \mathcal{M}^{*}} \sum_{k \in \mathcal{K}_{m}} R_{m, k}^{E 2 E}\left(\boldsymbol{\varphi}_{0}, \boldsymbol{\varphi}_{M}\right) \frac{1}{\theta_{m, k}}, \\
& \vartheta_{\text {tot }}\left(\boldsymbol{\varphi}_{0}, \boldsymbol{\varphi}_{M}, \boldsymbol{\theta}_{M}\right)=\eta_{0} P_{0} \sum_{m \in \mathcal{M}^{*}} \sum_{k \in \mathcal{K}_{m}} \frac{1}{\varphi_{0, m}} \frac{1}{\theta_{m, k}} \\
& +\sum_{m \in \mathcal{M}^{*}}\left(t_{m}^{\text {ope }}\left(\boldsymbol{q}_{m}, \boldsymbol{\theta}_{M}\right) P_{m}^{\text {fly }}+\eta_{m} P_{m} \sum_{k \in \mathcal{K}_{m}} \frac{1}{\varphi_{m, k}} \frac{1}{\theta_{m, k}}\right) .
\end{aligned}
$$

Note that $t_{m, k}^{c o n}, t_{G, m}^{f l y}$ and $t_{m, G}^{f l y}$ can be estimated when the clustering algorithm is implemented in the UAV deployment phase. Hence, for time allocation of UAV communications, we only focus on the data transferring time $t_{m, k}^{d a t}$ or $\theta_{m, k}$. However, the problem (26) is still a non-convex problem with the nonconvexity functions of (26a) and (26d). To solve problem (26), we use some efficient approximation approaches and logarithmic inequalities as in [24], [25] (see Appendix A). 
Firstly, we take

$$
\begin{gathered}
x_{1}=\frac{\varphi_{0, m}}{P_{0} \gamma_{0, m, m}}, \quad y_{1}=\sum_{m^{\prime} \in \mathcal{M}^{*} \backslash\{m\}} \frac{P_{0} \gamma_{0, m, m^{\prime}}}{\varphi_{0, m^{\prime}}}+\sigma_{m}^{2}, \\
\bar{x}_{1}=x_{1}^{(i)}=\frac{\varphi_{0, m}^{(i)}}{P_{0} \gamma_{0, m, m}} \\
\bar{y}_{1}=y_{1}^{(i)}=\sum_{m^{\prime} \in \mathcal{M}^{*} \backslash\{m\}} \frac{P_{0} \gamma_{0, m, m^{\prime}}}{\varphi_{0, m^{\prime}}^{(i)}}+\sigma_{m}^{2}, \\
x_{2}=\frac{\varphi_{m, k}}{P_{m}\left|g_{m, k}\right|^{2}}, \quad y_{2}=\sum_{l \in \mathcal{N}_{m}} \sum_{j \in \mathcal{K}_{l}} \frac{\beta_{l, k} P_{l}}{\varphi_{l, j}}+\sigma_{m, k}^{2}, \\
\bar{x}_{2}=x_{2}^{(i)}=\frac{\varphi_{m, k}^{(i)}}{P_{m}\left|g_{m, k}\right|^{2}}, \\
\bar{y}_{2}=y_{2}^{(i)}=\sum_{l \in \mathcal{N}_{m}} \sum_{j \in \mathcal{K}_{l}} \frac{\beta_{l, k} P_{l}}{\varphi_{l, j}^{(i)}}+\sigma_{m, k}^{2}, \\
t=\theta_{m, k}, \quad \bar{t}=t^{(i)}=\theta_{m, k}^{(i)} .
\end{gathered}
$$

By applying inequality (47) in the Appendix A, the $m$ th UAV's throughput and UE $(m, k)$ 's throughput can be approximated as

$$
R_{0, m}\left(\boldsymbol{\varphi}_{0}\right) \frac{1}{\theta_{m, k}} \geq \hat{D}_{0, m}^{(i)}\left(\boldsymbol{\varphi}_{0}, \boldsymbol{\theta}_{M}\right), \forall m \in \mathcal{M}^{*},
$$

where

$$
\begin{array}{r}
\hat{D}_{0, m}^{(i)}\left(\boldsymbol{\varphi}_{0}, \boldsymbol{\theta}_{M}\right)=\frac{B}{2}\left[\frac{2}{t^{(i)}} \ln \left(1+\frac{1}{x_{1}^{(i)} y_{1}^{(i)}}\right)\right. \\
+\frac{2}{t^{(i)}\left(x_{1}^{(i)} y_{1}^{(i)}+1\right)}-\frac{1}{t^{(i)} x_{1}^{(i)}\left(x_{1}^{(i)} y_{1}^{(i)}+1\right)} x_{1} \\
\left.-\frac{1}{t^{(i)} y_{1}^{(i)}\left(x_{1}^{(i)} y_{1}^{(i)}+1\right)} y_{1}-\frac{\ln \left(1+1 / x_{1}^{(i)} y_{1}^{(i)}\right)}{\left(t^{(i)}\right)^{2}} t\right],
\end{array}
$$

and

$$
R_{m, k}\left(\boldsymbol{\varphi}_{M}\right) \frac{1}{\theta_{m, k}} \geq \hat{D}_{m, k}^{(i)}\left(\boldsymbol{\varphi}_{M}, \boldsymbol{\theta}_{M}\right), \forall m \in \mathcal{M}^{*},
$$

where

$$
\begin{array}{r}
\hat{D}_{m, k}^{(i)}\left(\boldsymbol{\varphi}_{M}, \boldsymbol{\theta}_{M}\right)=\frac{B}{2}\left[\frac{2}{t^{(i)}} \ln \left(1+\frac{1}{x_{2}^{(i)} y_{2}^{(i)}}\right)\right. \\
+\frac{2}{t^{(i)}\left(x_{2}^{(i)} y_{2}^{(i)}+1\right)}-\frac{1}{t^{(i)} x_{2}^{(i)}\left(x_{2}^{(i)} y_{2}^{(i)}+1\right)} x_{2} \\
\left.-\frac{1}{t^{(i)} y_{2}^{(i)}\left(x_{2}^{(i)} y_{2}^{(i)}+1\right)} y_{2}-\frac{\ln \left(1+1 / x_{2}^{(i)} y_{2}^{(i)}\right)}{\left(t^{(i)}\right)^{2}} t\right] .
\end{array}
$$

Then,

$D_{m, k}^{E 2 E}\left(\boldsymbol{\varphi}_{0}, \boldsymbol{\varphi}_{M}, \boldsymbol{\theta}_{M}\right) \geq\left(D_{m, k}^{E 2 E}\right)^{(i)}\left(\boldsymbol{\varphi}_{0}, \boldsymbol{\varphi}_{M}, \boldsymbol{\theta}_{M}\right), \forall m \in \mathcal{M}^{*}$,

where

$$
\begin{aligned}
& \left(D_{m, k}^{E 2 E}\right)^{(i)}\left(\boldsymbol{\varphi}_{0}, \boldsymbol{\varphi}_{M}, \boldsymbol{\theta}_{M}\right) \\
& =\min \left(\hat{D}_{0, m}^{(i)}\left(\boldsymbol{\varphi}_{0}, \boldsymbol{\theta}_{M}\right), \hat{D}_{m, k}^{(i)}\left(\boldsymbol{\varphi}_{M}, \boldsymbol{\theta}_{M}\right)\right) .
\end{aligned}
$$

Finally, the total transmission data can be approximated as $D_{\text {tot }}^{E 2 E}\left(\boldsymbol{\varphi}_{0}, \boldsymbol{\varphi}_{M}, \boldsymbol{\theta}_{M}\right) \geq \sum_{m \in \mathcal{M}^{*}} \sum_{k \in \mathcal{K}_{m}}\left(D_{m, k}^{E 2 E}\right)^{(i)}\left(\boldsymbol{\varphi}_{0}, \boldsymbol{\varphi}_{M}, \boldsymbol{\theta}_{M}\right)$.
By changing the variable as (25), $\vartheta_{\text {tot }}\left(\boldsymbol{\varphi}_{0}, \boldsymbol{\varphi}_{M}, \boldsymbol{\theta}_{M}\right)$ in (27) is a convex function. Hence, given feasible points $\left(\varphi_{0}^{(i)}, \varphi_{M}^{(i)}\right.$, $\left.\boldsymbol{\theta}_{M}^{(i)}\right)$, we can achieve

$$
\eta^{(i)}=\sum_{m \in \mathcal{M}^{*}} \sum_{k \in \mathcal{K}_{m}} \frac{\left(D_{m, k}^{E 2 E}\right)^{(i)}\left(\boldsymbol{\varphi}_{0}^{(i)}, \boldsymbol{\varphi}_{M}^{(i)}, \boldsymbol{\theta}_{M}^{(i)}\right)}{\vartheta_{t o t}\left(\boldsymbol{\varphi}_{0}^{(i)}, \boldsymbol{\varphi}_{M}^{(i)}, \boldsymbol{\theta}_{M}^{(i)}\right)} .
$$

At the $i$ th iteration, the following convex program is solved to generate the next feasible point as follows:

$$
\begin{array}{r}
\max _{\boldsymbol{\varphi}_{0}, \boldsymbol{\varphi}_{M}, \boldsymbol{\theta}_{M}} \sum_{m \in \mathcal{M}^{*}} \sum_{k \in \mathcal{K}_{m}}\left(\left(D_{m, k}^{E 2 E}\right)^{(i)}\left(\boldsymbol{\varphi}_{0}, \boldsymbol{\varphi}_{M}, \boldsymbol{\theta}_{M}\right)\right. \\
\left.-\eta^{(i)} \vartheta_{t o t}\left(\boldsymbol{\varphi}_{0}, \boldsymbol{\varphi}_{M}, \boldsymbol{\theta}_{M}\right)\right)
\end{array}
$$

subject to $(26 b),(26 c)$,

$$
\begin{aligned}
& P_{0} \gamma_{0, m, m}\left(\frac{2}{\varphi_{0, m}^{(i)}}-\frac{\varphi_{0, m}}{\left(\varphi_{0, m}^{(i)}\right)^{2}}\right) \geq \\
& \left(e^{2 \bar{r}_{0, m} / B}-1\right)\left(\sum_{m^{\prime} \in \mathcal{M}^{*} \backslash\{m\}} \frac{P_{0} \gamma_{0, m, m^{\prime}}}{\varphi_{0, m^{\prime}}}+\sigma_{m}^{2}\right), \\
& m \in \mathcal{M}^{*},
\end{aligned}
$$

where the constraint (30c) is rewritten from the convex form of the constraint (26d).

The explicit formulation of (26d) can be represented as

$$
\begin{aligned}
& \frac{1}{2} B \ln \left(1+\frac{P_{0} \gamma_{0, m, m} / \varphi_{0, m}}{\sum_{m^{\prime} \in \mathcal{M}^{*} \backslash\{m\}} P_{0} \gamma_{0, m, m^{\prime}} / \varphi_{0, m^{\prime}}+\sigma_{m}^{2}}\right) \geq \bar{r}_{0, m}, \\
& m \in \mathcal{M}^{*}
\end{aligned}
$$

then

$$
\frac{P_{0} \gamma_{0, m, m}}{\varphi_{0, m}} \geq\left(e^{2 \bar{r}_{0, m} / B}-1\right)\left(\sum_{m^{\prime} \in \mathcal{M}^{*} \backslash\{m\}} \frac{P_{0} \gamma_{0, m, m^{\prime}}}{\varphi_{0, m^{\prime}}}+\sigma_{m}^{2}\right),
$$

$m \in \mathcal{M}^{*}$.

Then, we apply (45) in the Appendix A for the term of $\frac{1}{\varphi_{0, m}}$ in (32). Let us denote $(i)$ as the $i$ th iteration, the constraint (26d) can be rewritten as a convexity form as (30c).

Based on [26], the computational complexity of (30) is

$$
\mathcal{O}\left(\bar{n}^{2} \bar{m}^{2.5}+\bar{m}^{2.5}\right)
$$

with $\bar{n}=M(2 K+1)$, which is the number of the decision variables, and $\bar{m}=3 M+1$, which is the number of constraints.

We now proceed by proposing an algorithm to solve the EE maximisation problem (30). 
$\overline{\text { Algorithm } 2 \text { : Joint power and time transferring allocation }}$ procedure for solving problem (24)

Input: Set $i=0$ and a feasible point $\left(\boldsymbol{\varphi}_{0}^{(0)}, \boldsymbol{\varphi}_{M}^{(0)}, \boldsymbol{\theta}_{M}^{(0)}\right)$. Set the tolerance $\varepsilon=10^{-3}$ and the maximum number of iterations $I_{\max }=20$.

Calculate $\eta^{(0)}=\sum_{m \in \mathcal{M}^{*}} \sum_{k \in \mathcal{K}_{m}}\left(D_{m, k}^{E 2 E}\right)^{(i)}\left(\boldsymbol{\varphi}_{0}^{(0)}, \boldsymbol{\varphi}_{M}^{(0)}, \boldsymbol{\theta}_{M}^{(0)}\right) /$ $\vartheta_{\text {tot }}\left(\boldsymbol{\varphi}_{0}^{(0)}, \boldsymbol{\varphi}_{M}^{(0)}, \boldsymbol{\theta}_{M}^{(0)}\right)$.

Repeat

Solve problem (30) for the next optimal solution $\left(\varphi_{0}^{(i+1)}\right.$, $\left.\boldsymbol{\varphi}_{M}^{(i+1)}, \boldsymbol{\theta}_{M}^{(i+1)}\right)$.

Set $\eta^{(i+1)}=\sum_{m \in \mathcal{M}^{*}} \sum_{k \in \mathcal{K}_{m}}\left(D_{m, k}^{E 2 E}\right)^{(i+1)}\left(\boldsymbol{\varphi}_{0}^{(i+1)}, \boldsymbol{\varphi}_{M}^{(i+1)}\right.$, $\left.\boldsymbol{\theta}_{M}^{(i+1)}\right) / \vartheta_{t o t}\left(\boldsymbol{\varphi}_{0}^{(i+1)}, \boldsymbol{\varphi}_{M}^{(i+1)}, \boldsymbol{\theta}_{M}^{(i+1)}\right)$.

Set $i:=i+1$

Until Convergence of the objective function in (30) or $i>$ $I_{\max }$.

Output: $\left\{\boldsymbol{\varphi}_{0}^{*}, \boldsymbol{\varphi}_{M}^{*}, \boldsymbol{\theta}_{M}^{*}\right\}$ and $\left\{\eta^{*}\right\}$.

Note that the optimal solution of $\left\{\eta^{*}\right\}$ in (29) will be a suboptimal choice to maximise the EE formulated in (24) for the network.

The initial point $\left(\boldsymbol{p}_{0}^{(0)}, \boldsymbol{p}_{M}^{(0)},\left(\tau_{M}^{d a t}\right)^{(0)}\right)$ or $\left(\boldsymbol{\varphi}_{0}^{(0)}, \boldsymbol{\varphi}_{M}^{(0)}, \boldsymbol{\theta}_{M}^{(0)}\right)$ for (30) will be set up for satisfying constraints in the problem (26). The setting up initial point is by following a few rules as below.

- $\boldsymbol{p}_{0}^{(0)}$ can be found by solving the convex optimization problem as

$$
\begin{aligned}
\max _{\boldsymbol{p}_{0}>0} & \sum_{m \in \mathcal{M}^{*}} p_{0, m} \\
\text { subject to } & \sum_{m \in \mathcal{M}^{*}} p_{0, m} \leq 1, \\
& R_{0, m}\left(\boldsymbol{p}_{0}\right) \geq \bar{r}_{0, m}, \forall m \in \mathcal{M}^{*} .
\end{aligned}
$$

- $\boldsymbol{p}_{M}^{(0)}$ can be found by applying equal power allocation scheme as

$$
p_{m, k}=\frac{1}{K_{m}}, K_{m} \neq 0, \forall m \in \mathcal{M}^{*} .
$$

- $\left(\tau_{M}^{d a t}\right)^{(0)}$ can be found by applying equal time allocation scheme as

$$
t_{m, k}^{d a t}=\frac{\hat{T}_{m}^{\max }}{K_{m}}, K_{m} \neq 0, \forall m \in \mathcal{M}^{*} .
$$

where $\hat{T}_{m}^{\max }=T_{m}^{\max }-\left(\sum_{k \in \mathcal{K}_{m}} t_{m, k}^{c o n}+t_{G, m}^{f l y}+t_{m, G}^{f l y}\right)$.

\section{B. A central model for enhancing network EE performance with fairness services (CEE-QoS)}

In this sub-section, we are interested in a central model for enhancing network EE performance with fairness services (CEE-QoS). Here, we investigate the best-effort service for disaster emergency communications by applying UAV technology. For the QoS fairness, the main target is to maximise the minimum throughput in each cluster (max-min problem) by joint time-power allocation. This scheme can guarantee the
QoS for UEs located in the disaster areas. In addition, for the sake of comparison, we will plot the CEE-QoS performance with respect to the EE in Section 6 of simulation results. To this end, we develop a new Subproblem III from the Subproblem II as follows:

$$
\begin{array}{rr}
\multicolumn{3}{c}{\text { Subproblem III - A : }} \\
\max _{\boldsymbol{\varphi}_{0}, \boldsymbol{\varphi}_{M}, \boldsymbol{\theta}_{M}} \min ^{\forall m \in \mathcal{M}^{*}, \forall k \in \mathcal{K}_{m}} & D_{m, k}^{E 2 E}\left(\boldsymbol{\varphi}_{0}, \boldsymbol{\varphi}_{M}, \boldsymbol{\theta}_{M}\right) \\
\text { subject to } & (26 b),(26 c),(26 d),
\end{array}
$$

where the objective function is expressed as

$$
\begin{aligned}
& D_{m, k}^{E 2 E}\left(\boldsymbol{\varphi}_{0}, \boldsymbol{\varphi}_{M}, \boldsymbol{\theta}_{M}\right)=\frac{R_{m, k}^{E 2 E}\left(\boldsymbol{\varphi}_{0}, \boldsymbol{\varphi}_{M}\right)}{\theta_{m, k}} \\
& =\min \left\{\frac{R_{0, m}\left(\boldsymbol{\varphi}_{0}\right)}{\theta_{m, k}}, \frac{R_{m, k}\left(\boldsymbol{\varphi}_{M}\right)}{\theta_{m, k}}\right\}, \forall m, k .
\end{aligned}
$$

By applying optimisation techniques and Algorithm 2 in Section IV-A, the following max-min convex program is generated at the $i$ th iteration:

$$
\begin{array}{ll}
\max _{\boldsymbol{\varphi}_{0}, \boldsymbol{\varphi}_{M}, \boldsymbol{\theta}_{M}} & \min _{m \in \mathcal{M}^{*}, \forall k \in \mathcal{K}_{m}}\left(D_{m, k}^{E 2 E}\right)^{(i)}\left(\boldsymbol{\varphi}_{0}, \boldsymbol{\varphi}_{M}, \boldsymbol{\theta}_{M}\right) \\
\text { subject to } & (26 b),(26 c),(30 c) .
\end{array}
$$

We assume that $\left\{\varphi_{0}^{*}, \varphi_{M}^{*}, \theta_{M}^{*}\right\}$ is the solution of the problem (37). Thus, the EE performance can be calculated by using (26a).

The computational complexity of (37) is based on (33) with $\bar{n}=M(2 K+1)$ and $\bar{m}=3 M+1$.

\section{UAV-ENABLED DISASTER EMERGENCY COMMUNICATIONS VIA DISTRIBUTED RESOURCE ALLOCATION}

The centralised resource allocation approaches proposed in the previous section require a full coordination and cooperation between BS and all UAVs. Then, optimisation problem can be executed at Cloud Radio Access Network (CRAN). With this centralised strategy, when the network topology becomes very large, e.g., large number of users, the traffic load for data exchange at the BS and UAVs and the execution time for solving the optimisation problems at CRAN significantly increase. In a dynamically changing environment, e.g., UAV communications, it is unrealistic to endure such a high traffic load and long delay. To alleviate this shortcoming we are interested in solving the optimisation problems using a decentralised approach. To be more specific, the computational burden for solving the optimisation of resource allocation now lies at the clusters where each UAV has a capability to optimise its cluster performance.

In this section, we investigate efficient distributed schemes for the resource allocation, which are expected to significantly reduce the execution time of optimisation problems compared to the centralised approach.

\section{A. A distributed model for maximising network EE with EE objective (DEE-EE)}

In this sub-section, we are interested in a distributed model for maximising network $\mathrm{EE}$ with $\mathrm{EE}$ objective (DEE-EE). 
Here, a distributed model for solving EE optimisation problem (26) is shown as

Subproblem II - B :

$$
\max _{\boldsymbol{\varphi}_{m}, \boldsymbol{\theta}_{m}} \frac{D_{t o t, m}^{E 2 E}\left(\boldsymbol{\varphi}_{0}^{\text {fixed }}, \boldsymbol{\varphi}_{m}, \boldsymbol{\theta}_{m}\right)}{\vartheta_{t o t, m}\left(\boldsymbol{\varphi}_{0}^{\text {fixed }}, \boldsymbol{\varphi}_{m}, \boldsymbol{\theta}_{m}\right)}, \forall m \in \mathcal{M}^{*}
$$$$
\text { subject to }(26 b),(26 c),(26 d)^{*} \text {. }
$$

Remark 1: For handling the constraint (26d) in distributed scheme, we investigate $(26 d)^{*}$ with the BS power control by exploiting a solution of (34).

Here, it is to verify that $R_{0, m}\left(\varphi_{0}^{\text {fixed }}\right)=\bar{r}_{0, m}, m \in \mathcal{M}^{*}$, where $\boldsymbol{\varphi}_{0}^{\text {fixed }}=\boldsymbol{\varphi}_{0}^{(0)}=\left[1 / p_{0, m}^{(0)}\right]_{m \in \mathcal{M}^{*}}$ is the solution of problem (34).

Remark 2: To apply the distributed resource allocation, we study the information throughput at the $(m, k)$ th UE of the $m$ th cluster in (40) independently with other clusters by using equal power control in the second phase. Specifically, for $\varphi_{m}=\frac{K_{m}}{P_{m}}, \forall m \in \mathcal{M}^{*}$, we have

$$
\begin{aligned}
& \boldsymbol{\varphi}_{M}^{\text {fixed }}=\left[\boldsymbol{\varphi}_{m}\right]_{m \in \mathcal{M}^{*}}, R_{m, k}\left(\boldsymbol{\varphi}_{m}\right) \\
& =\frac{1}{2} B \ln \left(1+\frac{P_{m}\left|g_{m, k}\right|^{2} 1 / \varphi_{m, k}}{\mathcal{I}_{m, k}^{\text {inter }}\left(\boldsymbol{\varphi}_{M}^{\text {fixed }}\right)+\sigma_{m, k}^{2}}\right), \forall k \in \mathcal{K}_{m} .
\end{aligned}
$$

This means that the inter-cluster interference functions in $R_{m, k}\left(\boldsymbol{\varphi}_{m}\right)$ is considered as a constant value, i.e.,

$$
\mathcal{I}_{m, k}^{\text {inter }}\left(\varphi_{M}^{\text {fixed }}\right)=\sum_{l \in \mathcal{N}_{m}} \sum_{j \in \mathcal{K}_{l}} \beta_{l, k} \frac{K_{l}}{P_{l}}, k \in \mathcal{K}_{m}, m \in \mathcal{M}^{*} .
$$

Then, we have

$$
\begin{aligned}
& D_{\text {tot }, m}^{E 2 E}\left(\boldsymbol{\varphi}_{0}^{\text {fixed }}, \boldsymbol{\varphi}_{m}, \boldsymbol{\theta}_{m}\right)=\sum_{k \in \mathcal{K}_{m}} R_{m, k}^{E 2 E}\left(\boldsymbol{\varphi}_{0}^{\text {fixed }}, \boldsymbol{\varphi}_{m}\right) \frac{1}{\theta_{m, k}} \\
& =\sum_{k \in \mathcal{K}_{m}} \min \left\{\bar{r}_{0, m}, R_{m, k}\left(\boldsymbol{\varphi}_{m}\right)\right\} \frac{1}{\theta_{m, k}} \\
& \vartheta_{\text {tot }, m}\left(\boldsymbol{\varphi}_{0}^{\text {fixed }}, \boldsymbol{\varphi}_{m}, \boldsymbol{\theta}_{m}\right)=\eta_{0} P_{0} \sum_{k \in \mathcal{K}_{m}} \frac{1}{\varphi_{0, m}^{\text {fixed }}} \frac{1}{\theta_{m, k}} \\
& +t_{m}^{\text {ope }}\left(\boldsymbol{q}_{m}, \boldsymbol{\theta}_{m}\right) P_{m}^{\text {fly }}+\eta_{m} P_{m} \sum_{k \in \mathcal{K}_{m}} \frac{1}{\varphi_{m, k}} \frac{1}{\theta_{m, k}} .
\end{aligned}
$$

As a result, the total network EE performance by using distributed resource allocation can be calculated as

$$
E E=\sum_{m \in \mathcal{M}^{*}} V_{1, m}^{*}
$$

where $V_{1, m}^{*}$ is a local solution of problem (38) for the $m$ th cluster. Note that $V_{1, m}^{*}$ can be found by applying Algorithm 2 for $\left(\boldsymbol{\varphi}_{0}^{\text {fixed }}, \boldsymbol{\varphi}_{m}, \boldsymbol{\theta}_{m}\right)$.

Therefore, the computational complexity of (38) using the distributed scheme is based on (33) with $\bar{n}=2 K$ and $\bar{m}=3$.

\section{B. A distributed model for enhancing network EE with fair- ness services (DEE-QoS)}

In this sub-section, we are interested in a distributed model for enhancing network EE with fairness services (DEE-QoS).
Here, a distributed model for solving max-min program (35) is expressed as

Subproblem III-A :

$$
\begin{aligned}
\max _{\boldsymbol{\varphi}_{m}, \boldsymbol{\theta}_{m}} \min _{k \in \mathcal{K}_{m}} & D_{m, k}^{E 2 E}\left(\boldsymbol{\varphi}_{0}^{\mathrm{fixed}}, \boldsymbol{\varphi}_{m}, \boldsymbol{\theta}_{m}\right), \forall m \in \mathcal{M}^{*} \\
\text { subject to } & (26 b),(26 c),(26 d)^{*} \text { for only } m .
\end{aligned}
$$

Similarly to IV-B, at the $i$ th iteration, the following max-min convex program under the distributed scheme is equivalent to

$$
\max _{\boldsymbol{\varphi}_{m}, \boldsymbol{\theta}_{m}} \min _{k \in \mathcal{K}_{m}}\left(D_{m, k}^{E 2 E}\right)^{(i)}\left(\boldsymbol{\varphi}_{0}^{\mathrm{fixed}}, \boldsymbol{\varphi}_{m}, \boldsymbol{\theta}_{m}\right), \forall m \in \mathcal{M}^{*}
$$

subject to $(26 b),(26 c),(26 d)^{*}$, for only $m$.

We assume that $\left\{\varphi_{0}^{\text {fixed }}, \boldsymbol{\varphi}_{m}^{*}, \boldsymbol{\theta}_{m}^{*}\right\}$ is the solution of the problem (44) for cluster $m$. Thus, the EE performance can be calculated by using (26a).

Similarly, the computational complexity of (44) using the distributed scheme is based on (33) with $\bar{n}=2 K$ and $\bar{m}=3$.

\section{Simulation RESUltS}

In this section, the performance of the considered network is evaluated by the embedded optimisation packages in programming languages, i.e., the CVXPY package in Python [27]. The computational platform is used for performing simulation is a PC with CPU @ 3.7GHz and 32GB memory. For simulation setting, we set the system parameters as: The safety area is a circle coverage with a radius of $500 \mathrm{~m}$. The disaster area is an extended circle coverage from the safety area with a radius up to $2000 \mathrm{~m}$. The location of the $\mathrm{BS}$ is $(0,0,30)$ while the locations of UEs are randomly distributed in disaster area. The limited altitude of the UAVs $\left(H_{U, \min }, H_{U, \max }\right)$ is $(20,300) \mathrm{m}$. The connecting time between a UAV and a UE is set to 2 seconds. The speed of the UAVs is assumed constant at $10 \mathrm{~m} / \mathrm{s}$. The maximum operating time of the UAV is 20 minutes. The path loss threshold is set to $\gamma_{Q o S}=110 \mathrm{~dB}$. The tolerance and maximum number of iterations for convergence of algorithms are $\varepsilon=10^{-2}$ and $N_{\max }=20$. The bandwidth and carrier frequency are $B=10 \mathrm{MHz}$ and $f_{c}=2 \mathrm{GHz}$. The BS transmit power for communication is set as $40 \mathrm{~W}$. The number of BS antennas is 128. Other channel parameters are set as in [23], [24]. Note that although we consider the max-min throughput in the optimisation problems of CEE-QoS and DEE-QOS, the simulation results for these two cases are plotted in EE performance. To highlight the advantage of our proposed methods, we compares our approaches, i.e., CEE-EE, CEE-QOS, DEE-EE, and DEE-QOS, with some conventional methods described as follows:

- CEPTA: Combining equal power and equal time transferring allocation under central computing scheme.

- CEPOTA: Combining equal power allocation and optimal time transferring allocation under central computing scheme. CEPOTA-EE and CEPOTA-QoS are corresponding to EE and max-min data packet optimisation problems. 
- COPETA: Combining optimal power allocation and equal time transferring allocation under central computing scheme. COPETA-EE and COPETA-QoS are corresponding to $\mathrm{EE}$ and max-min data packet optimisation problems.

In this section, as illustrative cases, we set the number of UEs in critical area at $K=30$ and $K=60$ UEs while the number of UAVs is maximum at $M_{\max }=10$ and $M_{\max }=20$, respectively. Practical examples of these scenarios are shown as Fig. 3 and Fig. 4. Specifically, Fig. 3a provides a scenario with random locations of $K=30$ UEs and Fig. $3 \mathrm{~b}$ shows the clustering results by deploying UAVs for the scenarios.

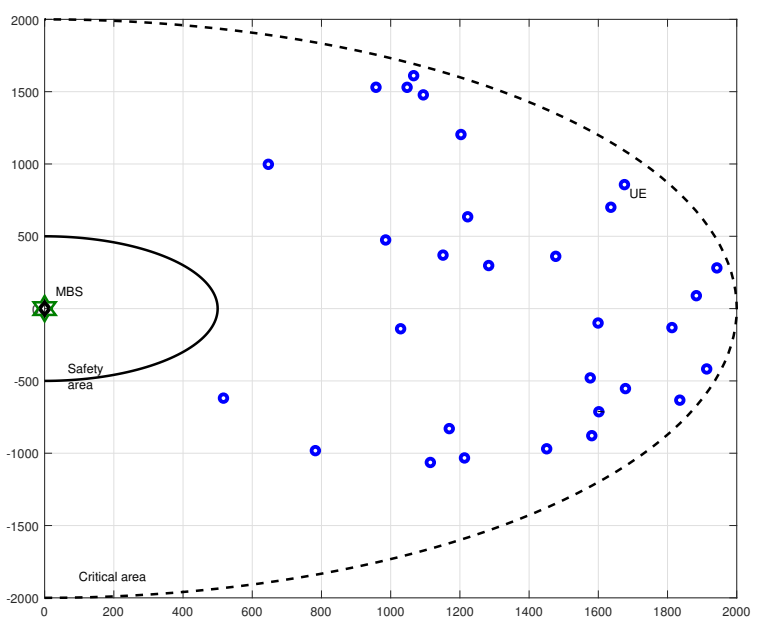

(a) Before clustering

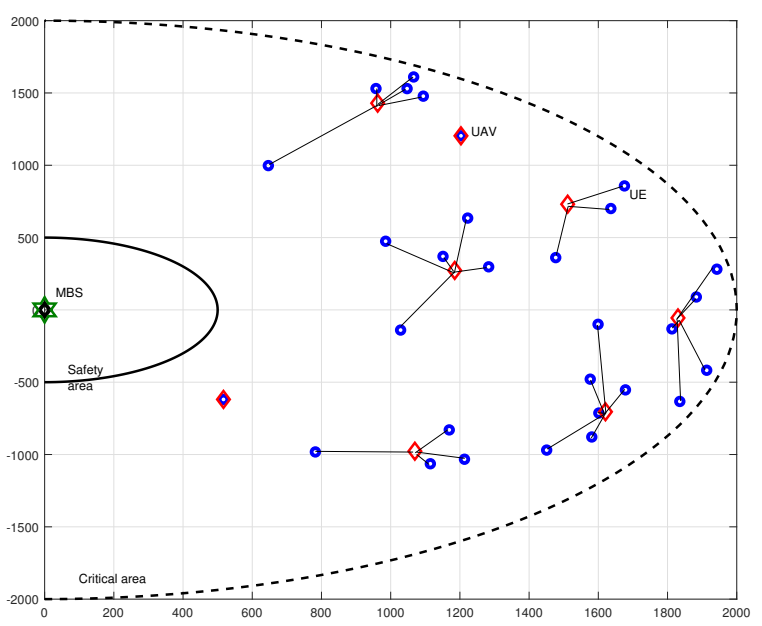

(b) After clustering. The number of UAVs used is 8 .

Fig. 3: A system model with $K=30$ UEs and $\gamma_{Q o S}=110 \mathrm{~dB}$ before and after the implementation of clustering algorithm.

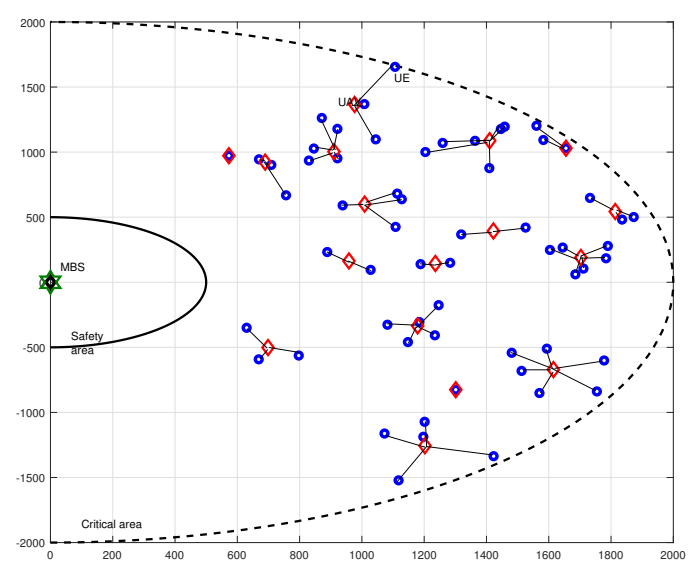

Fig. 4: A system model with $K=60$ UEs and $\gamma_{Q o S}=110$ $\mathrm{dB}$ for the implementation of clustering algorithm. After clustering, the number of UAVs used is 17 .

\section{A. Performance analysis of CEE-EE, CEE-QoS and CEPTA, CEPOTA, COPETA}

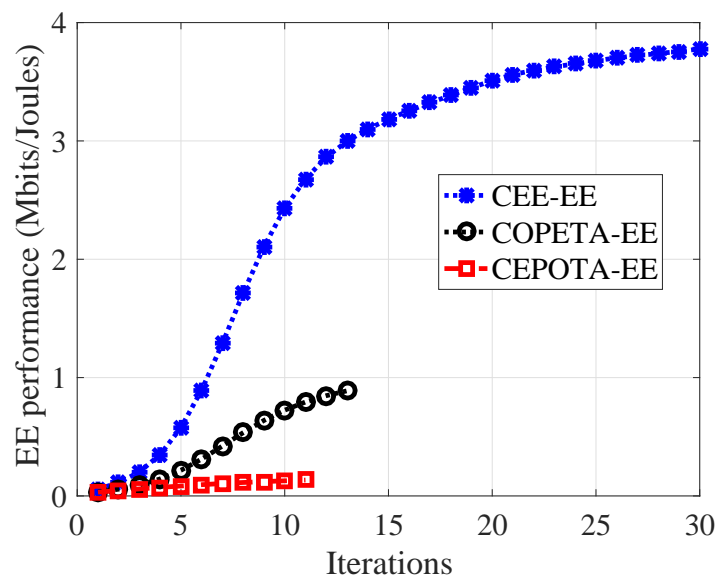

Fig. 5: The convergence of Algorithm 2 for solving the considered models: CEE-EE, CEPOTA-EE, COPETA-EE at $M=10, K=30$, and $P_{m}=1 W$.

In Fig. 5, we illustrate the convergence of Algorithm 2 for solving the CEE-EE, CEPOTA-EE, COPETA-EE models at $M=10, K=30$, and $P_{m}=1 W$. It is observed that after a few iterations, the objective function according to the three models converge to its maximum value. In particular, the CEEEE model requires significantly more iterations to converge when compared to the others due to the higher number of optimisation variables. The detailed analysis of the execution time for solving the considered problems is shown later in Section VI-B.

For EE performance of centralised approach, Fig. 6 plots the network EE performance for the proposed CEE-EE and CEE-QoS methods, the partial resource allocation methods (CEPOTA, COPETA) and the conventional method (CEPTA). 
As expected, CEE-EE provides a superior EE performance demonstrating the efficiency of joint power and time transferring allocation with EE objective function as shown in Fig. 6. Moreover, the EE performance of methods for optimising EE objective (CEPOTA-EE, COPETA-EE) obviously outperforms the other schemes (CEPTA, CEPOTA-QoS, COPETA-QoS). Considering network services under data packet achievement,

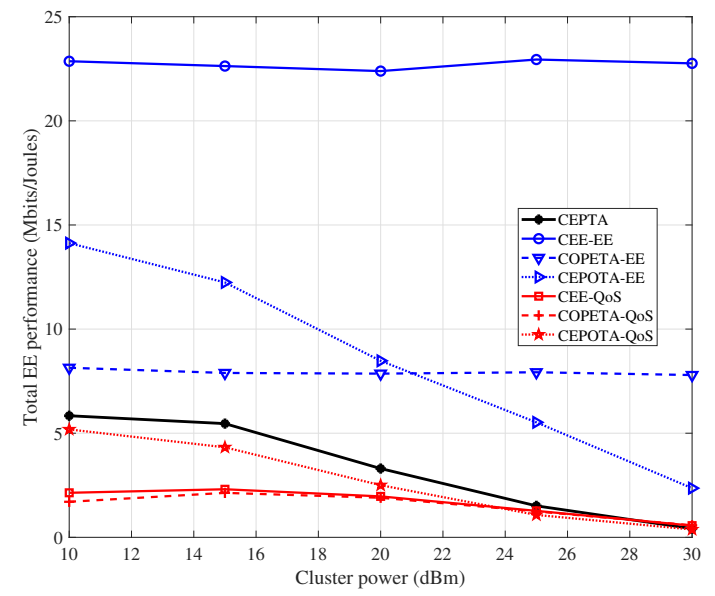

Fig. 6: Network EE performance for all the proposed methods under transmitted power at the UAV and the scenario of $K=$ 30 UEs.

we investigate the total network data and the worst UE data as below.

- The total network data is defined as the total received data (in bits) of $K$ UEs from the $M^{*}$ UAVs ( $M^{*}$ clusters) of the system.

- The worst UE data is defined as the total received data (in bits) of the UE among $K$ UEs with the lowest network service in the whole system.

As expected, a fairness of network service is guaranteed by applying optimal resource allocation under max-min optimisation (CEE-QoS, CEPOTA-QoS, COPETA-QoS). As shown in Fig. 8, CEE-QoS provides a superior network service for the UE with the lowest service compared to other scheme without optimal max-min throughput objective. In contrast, the total network data is highest in CEE-EE as shown in Fig. 7 since the transmitted packet is thoroughly enhanced in the CEE-EE approach. Consequently, the CEE-EE is not a good method for guaranteeing fairness service for all UEs as demonstrated in Fig. 8.

We also discuss the network service of weakest UE under max-min throughput approaches such as CEE-QoS, CEPOTAQoS,COPETA-QoS and the conventional method (CEPTA). The worst UE data gradually increases in the following order: CEPTA, CEPOTA-QoS, COPETA-QoS, CEE-QoS. Interestingly, the power control (COPETA-QoS) offers a better service than the time transferring control as shown in Fig. 10.

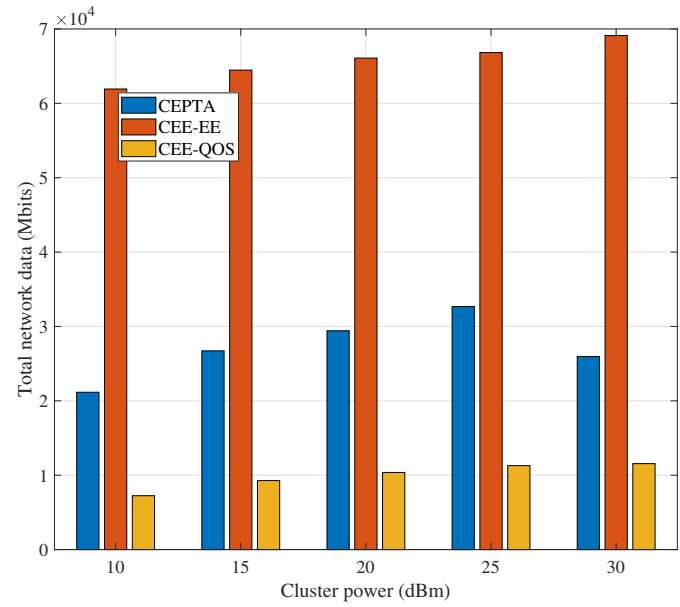

Fig. 7: Total network data for CEE-EE, CEE-QoS and CEPTA.

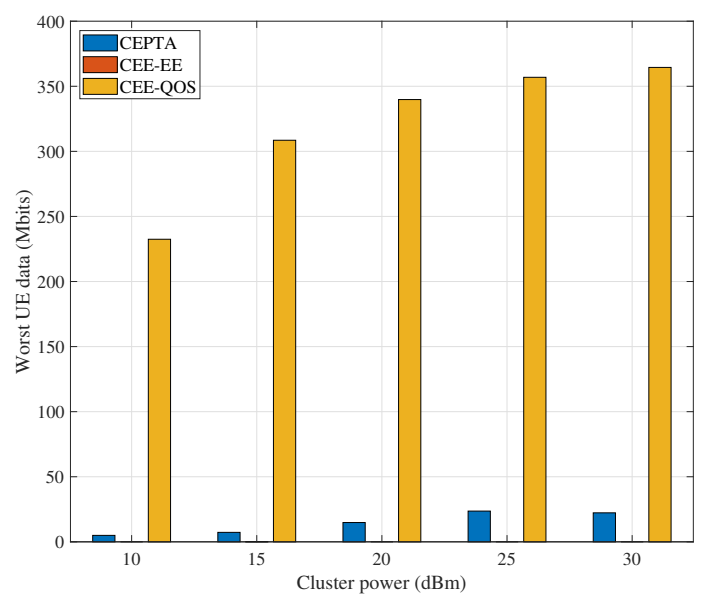

Fig. 8: Worst UE data for CEE-EE, CEE-QoS and CEPTA.

\section{B. Performance analysis of centralised and distributed re- source allocation}

For distributed scheme, we evaluate the total network data and the worst UE data as below.

- The total network data is defined as the total optimal data in every $M^{*}$ clusters.

- The worst UE data is defined as the total data of the UE with the lowest network service in the cluster providing the lowest service.

In Table I, we provide the average execution time for solving the problem (24), (35), (38) and (43) via all the proposed methods.

There is a tradeoff between the network performance and the execution time in centralised and distributed schemes for solving optimisation problems of resource allocation. As shown in Fig. 11 and Fig. 12, the network EE performance is significantly degraded in the centralised scheme (CEE-EE) and distributed scheme (DEE-EE). In contrast, we reduce a lot of time processing for solving resource allocation problems when DEE-EE method is implemented as analysed in Table I. 
TABLE I: Average execution time for solving optimisation problems via proposed method.

\begin{tabular}{|l|l|l|l|l|l|l|l|l|l|}
\hline Method & CEPTA & CEE-EE & DEE-EE & $\begin{array}{l}\text { CEPOTA- } \\
\text { EE }\end{array}$ & $\begin{array}{l}\text { COPETA- } \\
\text { EE }\end{array}$ & CEE-QoS & DEE-QoS & $\begin{array}{l}\text { CEPOTA- } \\
\text { QoS }\end{array}$ & $\begin{array}{l}\text { COPETA- } \\
\text { QoS }\end{array}$ \\
\hline $\begin{array}{l}\text { Execution } \\
\text { time }\end{array}$ & $34 \mathrm{~ms}$ & $25 \mathrm{~s}$ & $480 \mathrm{~ms}$ & $7 \mathrm{~s}$ & $11 \mathrm{~s}$ & $17 \mathrm{~s}$ & $280 \mathrm{~ms}$ & $4 \mathrm{~s}$ & $13 \mathrm{~s}$ \\
\hline
\end{tabular}

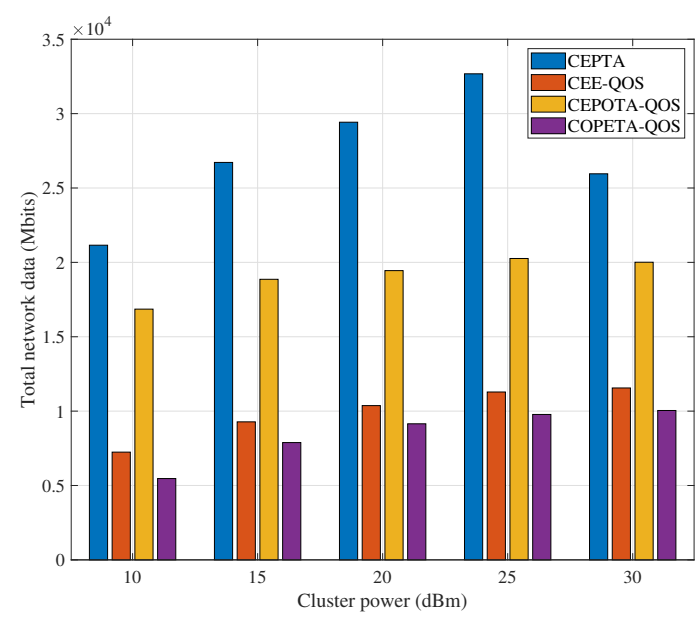

Fig. 9: Total network data for CEE-QoS and CEPTA, CEPOTA-QoS, COPETA-QoS.

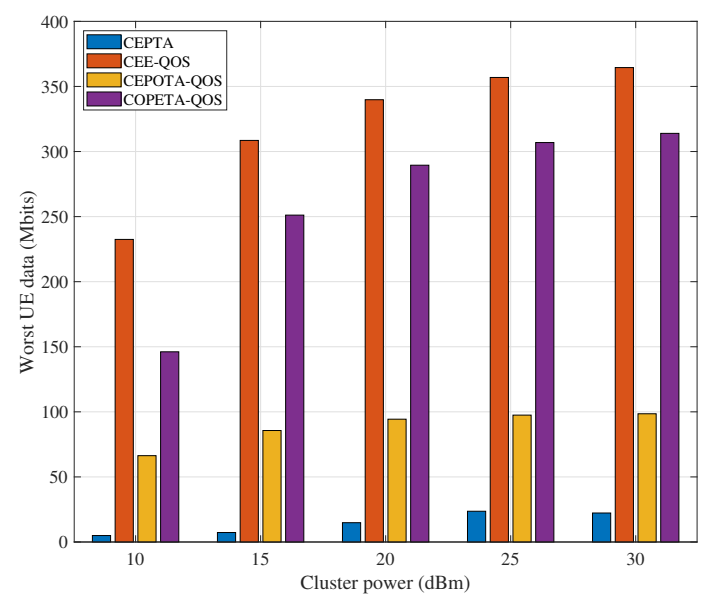

Fig. 10: Worst UE data for CEE-QoS and CEPTA, CEPOTAQoS, COPETA-QoS.

\section{Summary of results}

From all numerical results in this section, we can summary the method efficiency under system performance in terms of "Real-time", "Energy-Efficient", "QoS", "Low Energy" and "High Energy". where "Realtime": very small execution time, "Energy-Efficient": high EE performance, "QoS": fairness services for UEs, "Low Energy": low transmit power at UAV, "High Energy": high transmit power at UAV, and $\star$ : low priority, $\star \star$ : high priority.

\section{CONCLUSIONS}

We have investigated a downlink transmission of a massive MIMO BS and UAV-cellular relaying networks to meet the

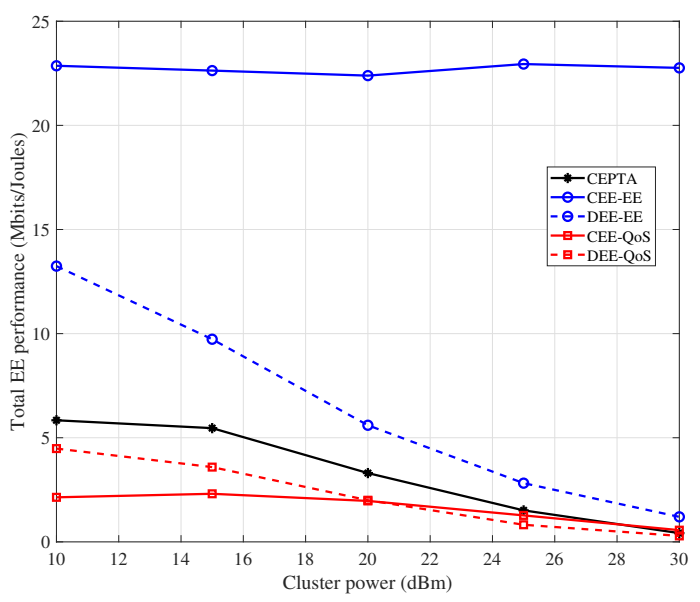

Fig. 11: Network EE performance between the centralised and distributed schemes under transmitted power at the UAV $\left(P_{m}\right)$ and $K=30$ UEs.

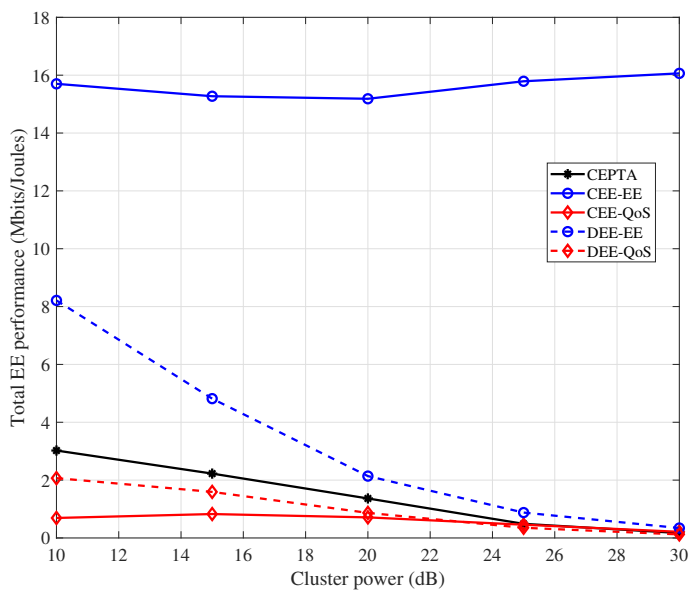

Fig. 12: Network EE performance between the centralised and distributed schemes under transmitted power at the UAV $\left(P_{m}\right)$ and $K=60$ UEs.

real-time service and stringent QoS constraints. We have proposed the joint optimisation framework for UAV clustering selection and resource allocation to maximise the network EE. We first considered the real-time UAVs deployment by proposing the K-means clustering algorithm. Then, the optimal resource allocation algorithms of low computational complexity with fast convergence were proposed in centralised and distributed manners. Numerical results were provided to demonstrate the advantage of the proposed approaches over the conventional schemes. 
TABLE II: Analysis of method efficiency.

\begin{tabular}{|c|c|c|c|c|c|}
\hline Method & Real-time & Energy-Efficient & QoS & Low Energy & High Energy \\
\hline CEPTA & $\star \star$ & & & & \\
\hline CEE-EE & & $\star \star$ & & $\star \star$ & $\star \star$ \\
\hline DEE-EE & $\star \star$ & $\star$ & & $\star \star$ & \\
\hline CEPOTA-EE & & $\star$ & & $\star$ & \\
\hline COPETA-EE & & $\star \star$ & & & \\
\hline CEE-QoS & & & $\star \star$ & $\star$ & $\star \star$ \\
\hline DEE-QoS & $\star \star$ & & $\star$ & $\star$ & \\
\hline CEPOTA-QoS & & & $\star$ & $\star$ & \\
\hline COPETA-QoS & & & $\star$ & & \\
\hline
\end{tabular}

\section{APPENDIX A}

\section{APPROXIMATION APPROACHES AND INEQUALITIES USED TO SOLVE PROBLEM (26)}

Firstly, we exploit an approximation technique for handling the nonconvexity of constraint (26d)

$$
\frac{1}{u} \geq \frac{2}{\bar{u}}-\frac{u}{\bar{u}^{2}}, \forall u>0, \bar{u}>0,
$$

where $\bar{u}>0$ is a given point. The constraint (26d) can be rewritten as a convexity form as shown in (30c)

$$
\begin{aligned}
& P_{0} \gamma_{0, m, m}\left(\frac{2}{\varphi_{0, m}^{(i)}}-\frac{\varphi_{0, m}}{\left(\varphi_{0, m}^{(i)}\right)^{2}}\right) \geq \\
& \left(e^{2 \bar{r}_{0, m} / B}-1\right)\left(\sum_{m^{\prime} \in \mathcal{M}^{*} \backslash\{m\}} \frac{P_{0} \gamma_{0, m, m^{\prime}}}{\varphi_{0, m^{\prime}}}+\sigma_{m}^{2}\right), m \in \mathcal{M}^{*} .
\end{aligned}
$$

Secondly, following the convexity of function $\frac{1}{t} \ln (1+$ $1 / x y)$, we exploit a logarithmic inequality for handling the objective function (26a) as below

$$
\frac{1}{t} \ln \left(1+\frac{1}{x y}\right) \geq a-b x-c y-d t,
$$

where

$$
\begin{gathered}
a=\frac{2}{\bar{t}} \ln \left(1+\frac{1}{\bar{x} \bar{y}}\right)+\frac{2}{\bar{t}(\bar{x} \bar{y}+1)}, b=\frac{1}{\bar{t} \bar{x}(\bar{x} \bar{y}+1)}, \\
c=\frac{1}{\bar{t} \bar{y}(\bar{x} \bar{y}+1)}, d=\frac{\ln (1+1 / \bar{x} \bar{y})}{\bar{t}^{2}}, \\
\forall t>0, \bar{t}>0, x>0, \bar{x}>0, y>0, \bar{y}>0 .
\end{gathered}
$$

Thus, the following nonconvex objective function (26a) is approximated into a convexity form as shown in (30a)

$$
\begin{array}{r}
\sum_{m \in \mathcal{M}^{*}} \sum_{k \in \mathcal{K}_{m}}\left(\left(D_{m, k}^{E 2 E}\right)^{(i)}\left(\boldsymbol{\varphi}_{0}, \boldsymbol{\varphi}_{M}, \boldsymbol{\theta}_{M}\right)\right. \\
\left.-\eta^{(i)} \vartheta_{t o t}\left(\boldsymbol{\varphi}_{0}, \boldsymbol{\varphi}_{M}, \boldsymbol{\theta}_{M}\right)\right) .
\end{array}
$$

\section{REFERENCES}

[1] L. D. Nguyen, K. K. Nguyen, A. Kortun, and T. Q. Duong, "Realtime deployment and resource allocation for distributed uav systems in disaster relief," in 2019 IEEE 20th International Workshop on Signal Processing Advances in Wireless Communications (SPAWC), Cannes, France, 2019, pp. 1-5.

[2] K. Mase, "How to deliver your message from/to a disaster area," IEEE Commun. Mag., vol. 49, no. 1, pp. 52-57, January 2011.
[3] D. G. C., A. Ladas, Y. A. Sambo, H. Pervaiz, C. Politis, and M. A. Imran, "An overview of post-disaster emergency communication systems in the future networks," IEEE Wireless Commun. Mag., vol. 26, no. 6, pp. 132-139, 2019.

[4] T. Q. Duong, L. D. Nguyen, and L. K. Nguyen, "Practical optimisation of path planning and completion time of data collection for uav-enabled disaster communications," in 2019 15th International Wireless Communications Mobile Computing Conference (IWCMC), Tangier, Morocco, 2019, pp. 372-377.

[5] A. Masaracchia, L. D. Nguyen, T. Q. Duong, and N. M. Nguyen, "An energy-efficient clustering and routing framework for disaster relief network," IEEE Access, vol. 7, pp. 56520-56 532, 2019.

[6] T. Taleb, Y. Hadjadj-Aoul, and T. Ahmed, "Challenges, opportunities, and solutions for converged satellite and terrestrial networks," IEEE Wireless Commun. Mag., vol. 18, no. 1, pp. 46-52, 2011.

[7] M. Mozaffari, W. Saad, M. Bennis, Y. Nam, and M. Debbah, "A tutorial on uavs for wireless networks: Applications, challenges, and open problems," IEEE Commun. Surveys Tuts., vol. 21, no. 3, pp. 23342360, 2019

[8] M. Nguyen, L. D. Nguyen, T. Q. Duong, and H. D. Tuan, "Realtime optimal resource allocation for embedded UAV communication systems," IEEE Wireless Communications Letters, vol. 8, no. 1, pp. 225228, Feb 2019.

[9] L. D. Nguyen, A. Kortun, and T. Q. Duong, "An introduction of real-time embedded optimisation programming for UAV systems under disaster communication," EAI Endorsed Transactions on Industrial Networks and Intelligent Systems, vol. 5, no. 17, 122018.

[10] Y. Zeng, R. Zhang, and T. J. Lim, "Wireless communications with unmanned aerial vehicles: opportunities and challenges," IEEE Commun. Mag., vol. 54, no. 5, pp. 36-42, 2016.

[11] N. Zhao, W. Lu, M. Sheng, Y. Chen, J. Tang, F. R. Yu, and K. Wong, "Uav-assisted emergency networks in disasters," IEEE Wireless Commun. Mag., vol. 26, no. 1, pp. 45-51, 2019.

[12] K. G. Panda, S. Das, D. Sen, and W. Arif, "Design and deployment of uav-aided post-disaster emergency network," IEEE Access, vol. 7, pp. $102985-102999,2019$

[13] M. Mozaffari, W. Saad, M. Bennis, and M. Debbah, "Efficient deployment of multiple unmanned aerial vehicles for optimal wireless coverage," IEEE Commun. Lett., vol. 20, no. 8, pp. 1647-1650, Aug 2016.

[14] X. Xi, X. Cao, P. Yang, J. Chen, T. Quek, and D. Wu, "Joint user association and uav location optimization for uav-aided communications," IEEE Wireless Commun. Lett., vol. 8, no. 6, pp. 1688-1691, 2019.

[15] Y. Zeng, R. Zhang, and T. J. Lim, "Throughput maximization for UAV-enabled mobile relaying systems," IEEE Trans. Commun., vol. 64, no. 12, pp. 4983-4996, Dec 2016.

[16] A. A. Nasir, H. D. Tuan, T. Q. Duong, and H. V. Poor, "UAV-enabled communication using NOMA," IEEE Trans. Commun., vol. 67, no. 7, pp. 5126-5138, 2019.

[17] S. Ahmed, M. Z. Chowdhury, and Y. M. Jang, "Energy-efficient uav relaying communications to serve ground nodes," IEEE Commun. Lett., vol. 24, no. 4, pp. 849-852, 2020.

[18] Y. Zeng, X. Xu, and R. Zhang, "Trajectory design for completion time minimization in UAV-enabled multicasting," IEEE Trans. Wireless Commun., vol. 17, no. 4, pp. 2233-2246, April 2018.

[19] K. Wagstaff, C. Cardie, S. Rogers, S. Schrödl et al., "Constrained K-means clustering with background knowledge," in Proceedings of the Eighteenth International Conference on Machine Learning, San Francisco, CA, USA, 2001, pp. 577-584.

[20] J. Mattingley and S. Boyd, "Real-time convex optimization in signal processing," IEEE Signal Process. Mag., vol. 27, no. 3, pp. 50-61, May 2010. 
[21] A. Al-Hourani, S. Kandeepan, and S. Lardner, "Optimal LAP altitude for maximum coverage," IEEE Wireless Commun. Lett., vol. 3, no. 6, pp. 569-572, Dec 2014.

[22] T. L. Marzetta, E. G. Larsson, H. Yang, and H. Q. Ngo, Fundamentals of massive MIMO. Cambridge University Press, 2016.

[23] M. Alzenad, A. El-Keyi, and H. Yanikomeroglu, "3-D placement of an unmanned aerial vehicle base station for maximum coverage of users with different QoS requirements," IEEE Wireless Commun. Lett., vol. 7, no. 1, pp. 38-41, Feb 2018.

[24] L. D. Nguyen, H. D. Tuan, T. Q. Duong, O. A. Dobre, and H. V. Poor, "Downlink beamforming for energy-efficient heterogeneous networks with massive MIMO and small cells," IEEE Trans. Wireless Commun., vol. 17, no. 5, pp. 3386-3400, May 2018.

[25] L. D. Nguyen, H. D. Tuan, T. Q. Duong, and H. V. Poor, "Multi-user regularized zero-forcing beamforming," IEEE Trans. Signal Process., vol. 67, no. 11, pp. 2839-2853, 2019.

[26] D. Peaucelle, D. Henrion, and Y. Labit, "Users guide for SeDuMi interface 1.03," 2002. [Online]. Available: http://homepages.laas.fr/ peaucell/software/sdmguide.pdf

[27] S. Diamond and S. Boyd, "CVXPY: A Python-embedded modeling language for convex optimization,” Journal of Machine Learning Research, vol. 17 , no. 83 , pp. $1-5,2016$.

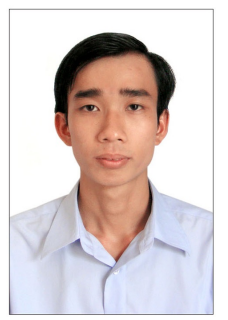

Tan Do-Duy received his B.S. degree from Ho Chi Minh City University of Technology (HCMUT), Vietnam, and M.S. degree from Kumoh National Institute of Technology, Korea, in 2010 and 2013, respectively. He received his Ph.D. degree from $\mathrm{Au}-$ tonomous University of Barcelona, Spain, in 2019. $\mathrm{He}$ is currently with the Department of Computer and Communication Engineering, Ho Chi Minh City University of Technology and Education (HCMUTE) in Vietnam as an Assistant Professor. His main research interests include wireless cooperative communications, real-time optimisation for resource allocation in wireless networks, and coding applications for wireless communications.

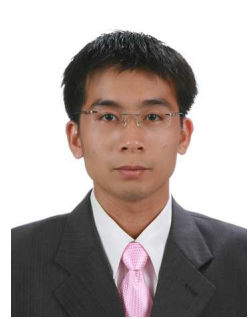

Trung Q. Duong (S'05, M'12, SM'13) is a Professor and Chair of Telecommunications at Queen's University Belfast (UK), where he was a Lecturer (Assistant Professor) (2013-2017), a Reader (Associate Professor) (2018-2020), and Full Professor from August 2020. He also holds a prestigious Research Chair of Royal Academy of Engineering. His current research interests include wireless communications, machine learning, realtime optimisation, and data analytic. He is the author or co-author of over $350+$ technical papers published in scientific journals (230+ articles) and presented at international conferences (140+ papers).

Dr. Duong currently serves as an Editor for the IEEE TRANSACTIONS ON WIRELESS COMMUNICATIONS and an Executive Editor for IEEE COMMUNICATIONS LetTERs. He was awarded the Best Paper Award at the IEEE Vehicular Technology Conference (VTC-Spring) in 2013, IEEE International Conference on Communications (ICC) 2014, IEEE Global Communications Conference (GLOBECOM) 2016 and 2019, IEEE Digital Signal Processing Conference (DSP) 2017, and International Wireless Communications \& Mobile Computing Conference (IWCMC) 2019. He is the recipient of prestigious Royal Academy of Engineering Research Fellowship (2015-2020) and has won a prestigious Newton Prize 2017.

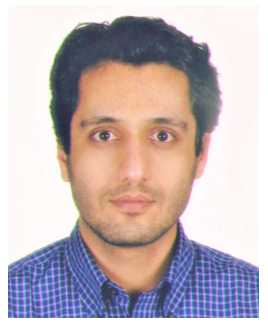

Saeed R. Khosravirad (Member, IEEE) received the B.Sc. degree from the Department of Electrical and Computer Engineering, University of Tehran, Iran, the M.Sc. degree from the Department of Electrical Engineering, Sharif University of Technology, Iran, and the Ph.D. degree in telecommunications from McGill University, Canada. From 2018 to 2019, he was with the Electrical and Computer Engineering Department, University of Toronto, Toronto, ON, Canada, as a Visiting Scholar. He has been with Bell Labs since 2015 . He is currently a member of Technical Staff with Nokia Bell Labs. His research interests include ultrareliable wireless communication for industrial Internet of Things and radio resource management for cellular technologies.

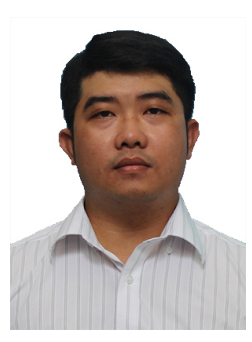

Long D. Nguyen (Member, IEEE) was born in Dong Nai, Vietnam. He received his B.S. degree in Electrical and Electronics Engineering and M.S. degree in Telecommunication Engineering from Ho Chi Minh City University of Technology (HCMUT), Vietnam, in 2013 and 2015, respectively. He received his Ph.D. degree in Electronics and Electrical Engineering from Queen's Univerisity Belfast (QUB), UK, in 2018. He is currently with Dong Nai University in Vietnam as an Assistant Professor and Duy Tan University as an Adjunct Assistant Professor. His research interests include convex optimization techniques and real-time embedded optimization for wireless communications and Internet of Things (IoTs).

He was awarded the Best Paper Award at the IEEE Digital Signal Processing (DSP) 2017, the IEEE International Conference on Recent Advances in Signal Processing, Telecommunication and Computing (Sigtelcom) 2018, the IEEE International Conference on Communications (ICC) 2019, the International Wireless Communications \& Mobile Computing Conference (IWCMC) 2019 and the IEEE Global Communications Conference (GLOBECOM) 2019. He was also awarded the Exemplary Reviewer Award in IEEE Communications Letters 2018.

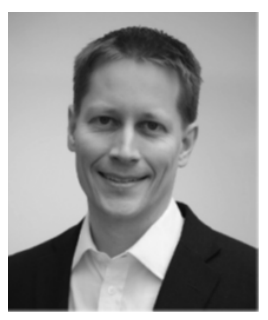

Holger Claussen (Senior Member, IEEE) received the Ph.D. degree in signal processing for digital communications from the University of Edinburgh, Edinburgh, U.K., in 2004. He is currently the Head of Wireless Communications Laboratory, Tyndall National Institute, Ireland. He was a Leader of the Wireless Communications Department, Nokia Bell Labs, Dubin, Ireland, and the USA. In this role, he and his team are innovating in all areas related to future evolution, deployment, and operation of wireless networks to enable exponential growth in mobile data traffic and reliable low latency communications. He joined Bell Labs, Murray Hill, NJ, USA, in 2004, where he began his research in the areas of network optimization, cellular architectures, and improving energy efficiency of networks. He is the author of one book, more than 125 publications, and 120 filed patent families. His research in this domain has been commercialized in Nokia's (formerly Alcatel-Lucent's) Small Cell product portfolio and continues to have significant impact. Dr. Claussen is a Fellow of the World Technology Network and the member of the Institution of Engineering and Technology (IET). He received the 2014 World Technology Award in the individual category communications technologies for innovative work of "the greatest likely long-term significance." Prior to this, he has been directing research in the areas of self-managing networks to enable the first large-scale femtocell deployments since 2009. 